\title{
Structural basis for neutralization of Plasmodium vivax by naturally acquired human antibodies that
} target DBP

\author{
Darya Urusova', Lenore Carias ${ }^{2}$, Yining Huang ${ }^{3,9}$, Vanessa C. Nicolete ${ }^{4}$, Jean Popovici', \\ Camille Roesch $^{5}$, Nichole D. Salinas ${ }^{6}$, Sebastien Dechavanne ${ }^{2}$, Benoit Witkowski ${ }^{5}$, \\ Marcelo U. Ferreira4, John H. Adams $\mathbb{D}^{7}$, Michael L. Gross $\mathbb{D}^{8}$, Christopher L. King ${ }^{2}$ and \\ Niraj H. Tolia ${ }^{1,6 \star}$
}

The Plasmodium vivax Duffy-binding protein (DBP) is a prime target of the protective immune response and a promising vaccine candidate for $P$. vivax malaria. Naturally acquired immunity (NAI) protects against malaria in adults residing in infectionendemic regions, and the passive transfer of malarial immunity confers protection. A vaccine that replicates NAI will effectively prevent disease. Here, we report the structures of DBP region II in complex with human-derived, neutralizing monoclonal antibodies obtained from an individual in a malaria-endemic area with NAI. We identified protective epitopes using X-ray crystallography, hydrogen-deuterium exchange mass spectrometry, mutational mapping and $P$. vivax invasion studies. These approaches reveal that naturally acquired human antibodies neutralize $P$. vivax by targeting the binding site for Duffy antigen receptor for chemokines (DARC) and the dimer interface of $P$. vivax DBP. Antibody binding is unaffected by polymorphisms in the vicinity of epitopes, suggesting that the antibodies have evolved to engage multiple polymorphic variants of DBP. The human antibody epitopes are broadly conserved and are distinct from previously defined epitopes for broadly conserved murine monoclonal antibodies. A library of globally conserved epitopes of neutralizing human antibodies offers possibilities for rational design of strain-transcending DBP-based vaccines and therapeutics against $P$. vivax.

M alaria remains a life-threatening disease causing high morbidity and mortality ${ }^{1}$. Despite the long history of human battle against malaria, a viable vaccine is still desperately needed but remains elusive. This is in spite of the recognition that adults in malaria-endemic areas develop relative immunity to malaria infection ${ }^{2-4}$. Further, passive transfer of $\gamma$-globulins isolated from serum of patients exposed to malaria to infected non-immune children less than five years of age, demonstrated a significant reduction in parasitaemia ${ }^{2,5-7}$. Defining the structural correlates of naturally acquired immunity (NAI) is a critical factor for the design of a universal vaccine.

The Plasmodium vivax Duffy-binding protein (DBP) is the most promising vaccine candidate for $P$. vivax malaria ${ }^{8-21}$. During reticulocyte invasion, $P$. vivax uses a Duffy-binding-like (DBL) domain in DBP, also known as region II (DBP-II) to engage Duffy antigen receptor for chemokines (DARC) on host reticulocytes ${ }^{8-12,14-18}$. DBP-II binds DARC via receptor-induced ligand dimerization, sandwiching DARC residues $19-30$ between two DBP-II molecules $^{17,18}$. DBP-II comprises three subdomains (1 to 3 ); subdomain 2 (SD2) is responsible for dimerization and receptor binding, which are required to engage DARC ${ }^{17,18}$. Rabbit and human antibodies that block the DBP-DARC interaction neutralize $P$. vivax, suggesting that a DBP-based vaccine will reduce infection ${ }^{5}$. However, the successful design of a DBP-II-based vaccine may be limited by strain-specific immune responses due to the polymorphic nature of $\mathrm{DBP}^{22,23}$ and the presence of immunodominant but non-protective epitopes within $\mathrm{DBP}^{24,25}$. Despite the polymorphic nature of DBP, broadly conserved epitopes of three inhibitory murine monoclonal antibodies have been identified in subdomain 3 of DBP- $\mathrm{II}^{19}$. These epitopes are distant from the dimer interface and DARC-binding site $^{19}$. Furthermore, human vaccination with DBP-II elicits antibodies that block in vitro binding of four alleles of DBP to DARC, suggesting that broadly neutralizing epitopes of human antibodies may exist within DBP-II ${ }^{20,21}$.

The identification of broadly conserved human neutralizing-antibody epitopes that contribute to naturally acquired immunity is essential for the improved rational design of potent strain-transcending DBP-based vaccines. Here we present the study of DBP-II in complex with two human neutralizing monoclonal antibodies, 053054 and 092096. These human monoclonal antibodies were produced by sorting individual DBP-II-specific B cells from a Cambodian donor with naturally acquired DBP-II-blocking antibodies and isolating, sequencing and cloning the variable regions from human $y$-immunoglobulin (IgG) heavy and light chains. Structures of DBP-II-antibody complexes were determined by X-ray crystallography, and epitopes were further mapped by hydrogen-deuterium exchange mass spectrometry (HDX-MS) and mutational studies. Both antibodies inhibit binding of DBP to red blood cells, and 092096 neutralizes $P$. vivax in ex vivo

'Department of Molecular Microbiology, Washington University School of Medicine, Saint Louis, MO, USA. ${ }^{2}$ Center for Global Health and Diseases, Case Western Reserve University, Cleveland, OH, USA. ${ }^{3}$ Department of Chemistry, Washington University in St Louis, St Louis, MO, USA. ${ }^{4}$ Department of Parasitology, University of Sao Paulo, Sao Paulo, Brazil. ${ }^{5}$ Malaria Molecular Epidemiology Unit, Pasteur Institute in Cambodia, Phnom Penh, Cambodia. ${ }^{6}$ Laboratory of Malaria Immunology and Vaccinology, National Institute of Allergy and Infectious Diseases, National Institutes of Health, Bethesda, MD, USA. 'Department of Global Health, College of Public Health, University of South Florida, Tampa, FL, USA. ${ }^{8}$ Department of Chemistry, Washington University in St Louis, St Louis, MO, USA. 'Present address: Bioproduct Research and Development, Lilly Research Laboratories, Eli Lilly and Company, Indianapolis, IN, USA. *e-mail: niraj.tolia@nih.gov 
experiments. Polysera from patient populations competes with binding of 092096 to DBP. We show that these naturally acquired human antibodies neutralize $P$. vivax by targeting the DARC-binding site and dimer interface of $P$. vivax $\mathrm{DBP}$. This work forms a strong foundation for the rational design of potent strain-transcending DBP-based vaccines against $P$. vivax.

\section{Results}

Isolation of human monoclonal antibodies 053054 and 092096. $I G H$ and IGL PCR products from 98 individual B cells from one Cambodian donor were sequenced, and $16 \mathrm{~B}$ cell clonal groups as defined by sequences of the variable (V) region of the IgG heavy chain with the same inferred $\mathrm{VH}_{\mathrm{H}}$ and $\mathrm{JH}$ germline sequences, identical CDR3 length, and the same or very similar CDR3 sequences. One or two clones were selected from each group and expressed as full-length IgG1 proteins, thereby creating monoclonal antibodies. Monoclonal antibodies from 11 clones recognized DBP-II. We selected one clone from each of two clonal groups corresponding to two of the larger clonal groups in terms of the number of DBP-IIspecific B cells isolated by single-cell sorting. These two monoclonal antibodies were designated 092096 and 053054.

Structures of human antibodies 053054 and 092096 in complex with DBP-II. We solved two crystal structures of DBP-II in complex with a single-chain variable fragment $(\mathrm{scFv})$ generated from the human monoclonal antibodies 053054 and 092096 (Fig. 1a,c and Supplementary Table 1). The electron density maps clearly define the contact sites of the DBP-II-antibody interface in both structures (Supplementary Fig. 1a,b). Both antibodies bind to the same face of DBP, although the orientation of the heavy and light chains relative to DBP differs (Supplementary Fig. 2). The interacting residues show a substantial overlap between the epitopes for the two antibodies (Supplementary Table 2).

The discontinuous conformational epitope for 053054 comprises residues D264-A281 and Q356-N372, situated in two outer helices of subdomain 2, and E249 of the N-terminal helix in subdomain 1 (Fig. 1a,b and Supplementary Table 2). Antibody 092096 binds to the discontinuous conformational epitope comprising residues L270-K289, A355-W375, E249 and Y219 (Fig. 1c,d and Supplementary Table 2). All complementarity-determining regions (CDRs) of both antibodies contact DBP (Supplementary Table 2), and the buried surface area and shape complementarity of the interactions are within standard parameters (Supplementary Fig. 1c,d). These epitopes differ from previously reported epitopes for broadly conserved inhibitory murine monoclonal antibodies located in subdomain 3 of DBP-II (Fig. 1e). The overlap of human epitopes in DBP coupled with distinct modes of binding indicate multiple pathways for antibody engagement of the surface comprising residues D264-K289 and Q356-W375 in DBP.

Mechanism of neutralization. Binding of 053054 or 092096 does not cause conformational changes within DBP-II as there are negligible structural differences between antibody-bound, unbound and DARC-bound DBP (Supplementary Table 3). However, structural comparison of DBP-II-053054 and DBP-II-092096 with the DBP-IIDARC complex reveals that 053054 and 092096 engage residues in the DARC-binding cleft and dimer interface of DBP-II (Fig. 2). Residues F261-T266, L270-K289 and Q356-K367 engage the DARC ectodomain, and residues F261-F267, L270-Y278 and E352-Q356 to form a dimer interface ${ }^{18}$. These segments overlap considerably with the epitopes for 053054 and 092096, suggesting that the antibody association prevents DBP dimerization and DARC binding (Fig. 2c,f).

Human antibodies 053054 and 092096 block DBP binding to RBCs and neutralize $P$. vivax invasion ex vivo. The structural studies suggest that the antibodies 053054 and 092096 directly block DBP binding to DARC on red blood cells and would therefore neutralize $P$. vivax merozoites. To test this hypothesis, we examined inhibition of DBP-II binding to red blood cells (RBCs) (Fig. 3a). Both human antibodies completely blocked the binding, with half-maximum inhibitory concentration $\left(\mathrm{IC}_{50}\right)$ values for 053054 and 092096 of $4.88 \pm 1.06 \mathrm{nM}$ and $1.63 \pm 1.07 \mathrm{nM}$, respectively (presented as mean \pm s.d.), whereas an isotype control, 043038, did not inhibit binding. Owing to its more potent inhibition of DBP-II binding to RBCs, 092096 was used in further experiments to test its ability to neutralize $P$. vivax invasion of human reticulocytes ex vivo. Using $P$. vivax-infected erythrocytes obtained from Brazilian patients, we showed that $100 \mu \mathrm{g} \mathrm{ml}^{-1}$ 092096 inhibits $P$. vivax invasion by $43 \%$ compared with no antibody $(P=0.0001)$ (Fig. 3b). We also performed the $P$. vivax ex vivo assay with clinical isolates of $P$. vivax from Cambodia to assess the ability of this antibody to neutralize parasites from distinct geographical locations. We found that $100 \mu \mathrm{g} \mathrm{ml}^{-1} 092096$ inhibits $P$. vivax invasion by $47.4 \%(P=0.002)$ and $500 \mu \mathrm{g} \mathrm{ml}^{-1} 092096$ inhibits invasion by $86.6 \%(P=0.0007)$ (Fig. $3 \mathrm{c})$. Note that the degree of invasion inhibition is similar for the different parasite isolates. This result indicates a single human monoclonal antibody that targets the receptor-binding residues and the dimer interface of DBP can neutralize invasion of multiple $P$. vivax isolates from distinct geographical locations.

Neutralizing epitopes are widely recognized in patient populations. We examined whether the epitope recognized by 092096 is widespread in patient populations exposed to P. vivax (Fig. 3d,e). Functional monoclonal antibodies found in individuals with high levels $(\geq 80 \%)$ of blocking antibody activity to DBP-II who resided in $P$. vivax-endemic regions of Cambodia recognize similar epitopes as 092096 (Fig. 3d). For example, the serum sample highlighted by the box in Fig. 3d blocks $100 \%$ of DBP-II binding to DARC N-terminus at a dilution of 1:20, and 092096 (dilution of $1: 50)$ competed for $90 \%$ of the blocking activity. The ability of 092096 and 087086 (a non-blocking antibody to DBP-II) to compete with polysera divided serum samples into two classes, those with $80-100 \%$ blocking activity versus those with $40-79 \%$ blocking activity (Fig. 3e). Monoclonal antibody 092096 demonstrated clear competition with high-blocking-activity polysera. These results demonstrate that the epitopes identified here are widely recognized by sera in patient populations.

Mutations and ELISA reveal overlapping binding sites with different binding requirements. The epitopes were evaluated using surface mutant libraries of DBP-II and enzyme-linked immunosorbency assay (ELISA). Both 053054 and 092096 recognized wildtype Sal-1 DBP-II and mutants 17 and 19, with substituted residues outside either epitope, equally well (Fig. 4a, and Supplementary Fig. 3). By contrast, 053054 did not bind to mutants 18 and 20, as they contain mutations in the epitope for 053054. Consistent with overlapping epitopes between the two monoclonal antibodies, 092096 also lost binding to mutant 20. Mutant 18, however, had no effect on binding to 092096 . This divergence in binding to mutant 18 is caused by interaction differences due to the altered orientation of heavy and light chains of 053054 and 092096 with respect to DBP (Fig. 4b,c and Supplementary Fig. 2). Although the residues substituted in mutant 18-F267, Y271, K274 and Y278are bound to the core of 053054 and interact with CDR3 on both the heavy and light chains (Fig. 4b), they only bind to the periphery of 092096 and predominantly interact with its light chain (Fig. 4c). On the contrary, residues Y363, K367 and K370, which are substituted in mutant 20 , interact with the CDR3 on the heavy chain in 092096 and contact the core of 053054 through CDRs 1 and 2 of its light chain. Therefore, this region of DBP is crucial for binding to both antibodies. 


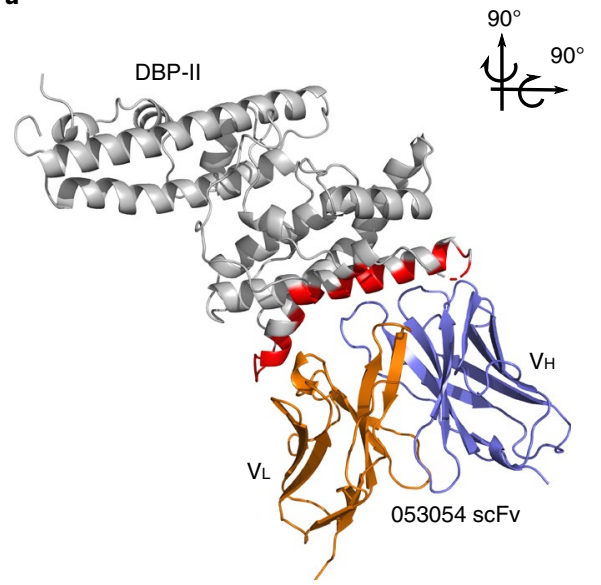

c

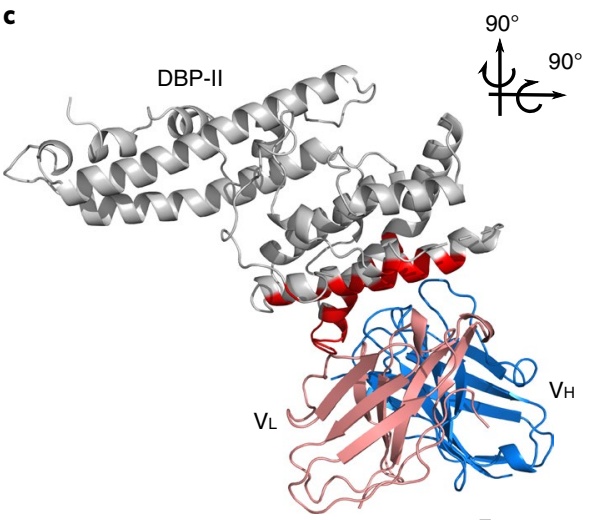

092096 scFv

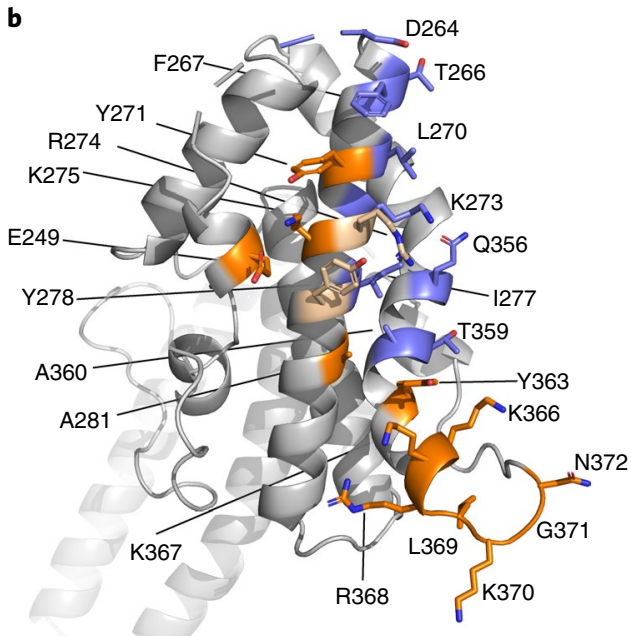

d

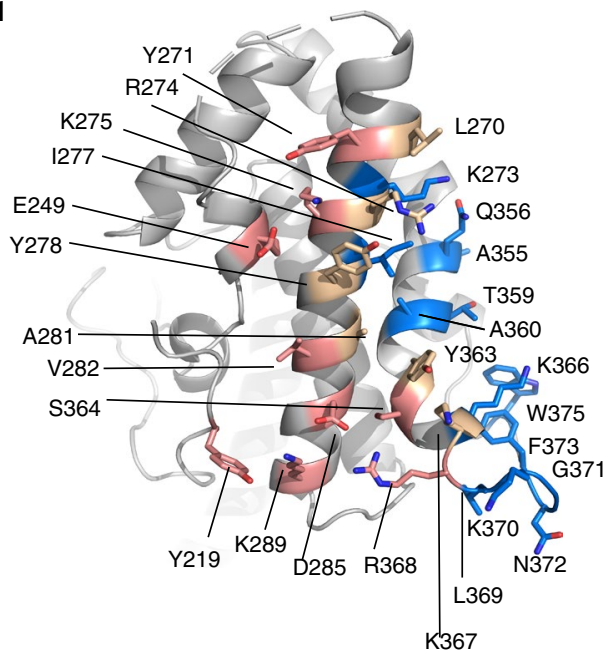

e

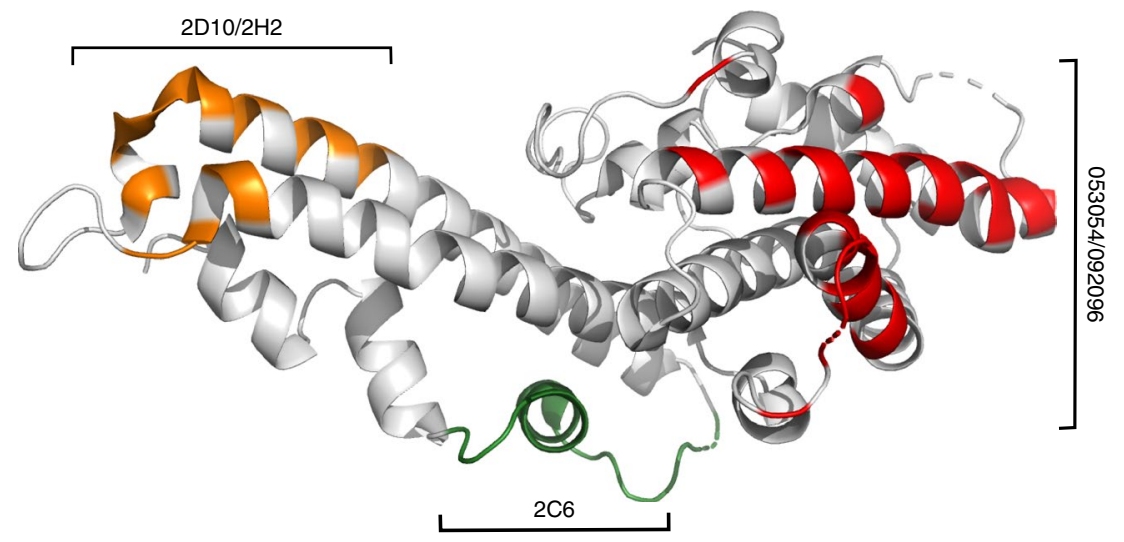

Fig. 1 Structural definition of human antibody epitopes in DBP. a, Overall structure and epitope for the DBP-053054 complex. Grey, DBP-II; dark blue, 053054 heavy chain; orange, 053054 light chain; red, epitope. b, Orthogonal detailed view of the DBP epitope for 053054. Grey, DBP-II; dark blue, DBP residues contacted by the 053054 heavy chain; orange, DBP residues contacted by the 053054 light chain; beige, DBP residues contacted by both heavy and light chains. c, Overall structure and epitope for the DBP-092096 complex. Grey, DBP-II; light blue, 092096 heavy chain; pink, 092096 light chain; red, epitope. d, Orthogonal detailed view of the DBP epitope for 092096. Grey, DBP-Il; light blue, DBP residues contacted by the 092096 heavy chain; pink, DBP residues contacted by the 092096 light chain; beige, DBP residues contacted by both heavy and light chains. e, Comparison of epitopes for human and mouse antibodies ${ }^{19}$ in DBP-II reveal distinct epitopes for each species. Orange, epitope for inhibitory murine monoclonal antibodies 2D10 and $2 \mathrm{H} 2$; green, epitope for inhibitory murine monoclonal antibody 2C6; red, epitopes of neutralizing human monoclonal antibodies 053054 and 092096.

HDX-MS determination of the epitope for 053054 and 092096. HDX-MS was used to independently assess the epitopes for monoclonal antibodies 053054 and 092096 in solution (Fig. 4d). In agree- ment with the structural data, peptide $268-281$ is significantly attenuated from exchange upon binding to 053054 , whereas no difference is detected for this peptide with 092096. Regions 288-298 

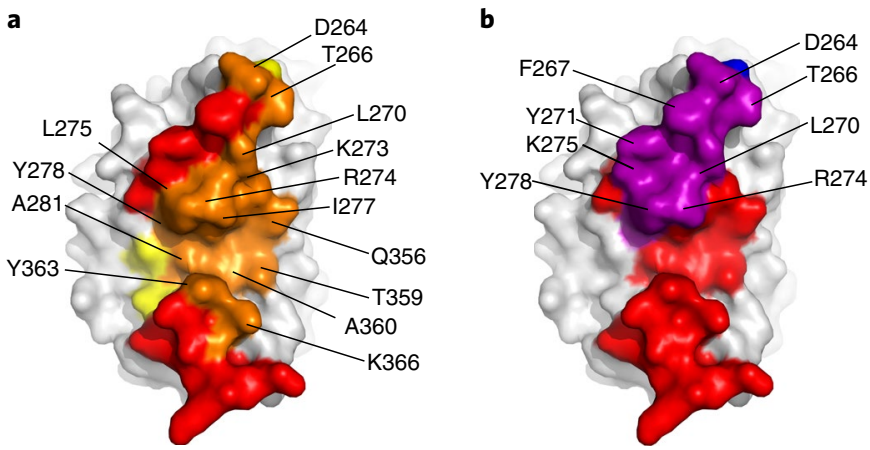

C

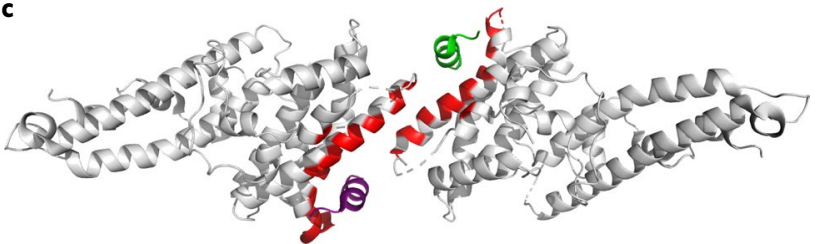

d
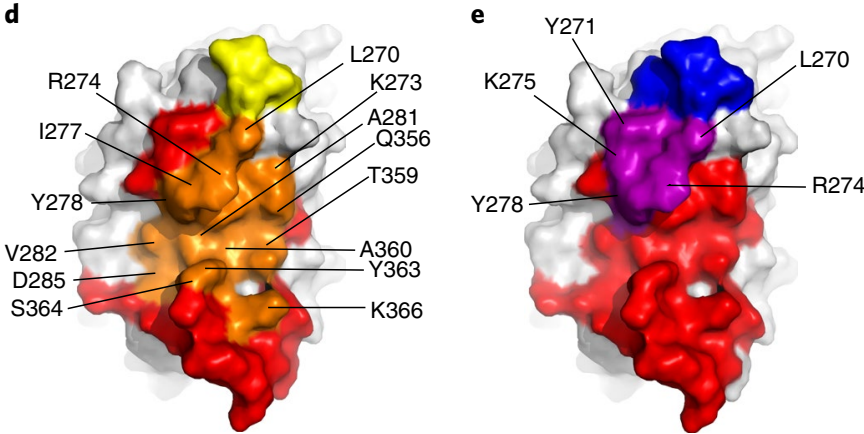

f

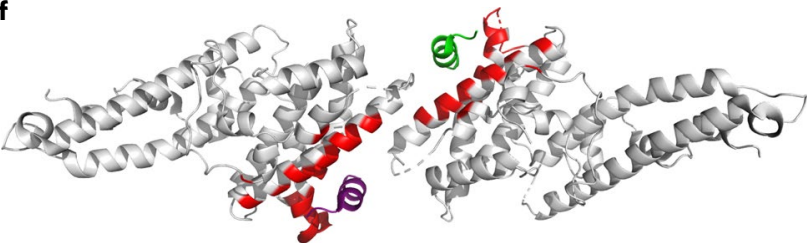

Fig. 2 | Neutralizing antibodies 053054 and 092096 block DARC-binding site and dimer interface. a, Overlay of the 053054 epitope with the DARCbinding residues in DBP. Orange, overlap between the 053054 epitope and the DARC binding site; yellow-orange, DARC-binding site; red-orange, the 053054 epitope. b, Overlay of 053054 epitope with the dimer interface of DBP. Purple, overlap between the 053054 epitope and the dimer interface; blue-purple, dimer interface; red-purple, the 053054 epitope. c, Mapping the 053054 epitope on the tetramer structure of DARC-bound DBP shows the epitope overlapping with the DARC-binding residues and dimer interface. Grey, DBP; green, DARC monomer 1; purple, DARC monomer 2; red, epitope of 053054. d, Overlay of the 092096 epitope with the DARCbinding residues in DBP. Orange, overlap between the 092096 epitope and the DARC-binding site; yellow-orange, DARC-binding site; red-orange, the 092096 epitope. e, Overlay of 092096 epitope with the dimer interface of DBP. Purple, overlap between the 092096 epitope and the dimer interface; blue-purple, dimer interface; red-purple, the 092096 epitope. f, Mapping the 092096 epitope on the tetramer structure of DARC-bound DBP shows the epitope overlapping with the DARC-binding residues and dimer interface. Grey, DBP; Green, DARC monomer 1; purple, DARC monomer 2; red, epitope of 092096.

and 364-379 show substantial HDX attenuation against 092096 predominantly on peptide 364-373. Peptide 364-373 also responds for 053054 but with less attenuation, and converging kinetics indicate weaker binding for 053054. By contrast, peptides 288-298 and 374-379 are silent with 053054 (Fig. 4d). The remaining peptides either show minor differences in deuterium exchange upon antibody binding or no difference, including the broadly conserved epitope for murine antibodies 2D10, $2 \mathrm{H} 2^{19}$ (Fig. $4 \mathrm{~d}$ and Supplementary Figs. 4 and 5).

Polymorphisms within or in the vicinity of the epitopes for 053054 and 092096 do not affect antibody binding to DBP. Having determined the human neutralizing epitopes and the mechanism of antibody neutralization, we examined DBP variation within the epitopes. We first determined affinities of 053054 and 092096 to the reference strain Sal-1 DBP using bio-layer interferometry (BLI) (Supplementary Table 4 and Supplementary Figs. 6 and 7). The steady-state equilibrium dissociation constants were $7.44 \pm 0.36 \mathrm{nM}$ for 053054 and $7.63 \pm 0.29 \mathrm{nM}$ for 092096 , respectively. BLI also provides the association and dissociation rates that can inform the half-life of antibody binding. In both cases, the dissociation rates were remarkably slow, indicating that antibody binding leads to a stable, long-lived complex. The slow off-rate is consistent with the HDX data that show no convergence of HDX kinetics at long exchange times. These results indicate that the two antibodies have comparable binding affinity.

A comparative alignment of 599 DBP sequences from diverse isolates of $P$. vivax revealed a number of polymorphisms within the epitopes for 053054 and 092096 and adjacent residues (Supplementary Table 5). The most common polymorphisms individually and in combination are R263S and N372K. Sal-1, the strain used to isolate and crystallize 053054 and 092096, contains R263 and N372 at these positions. R263 is located in a disordered segment at the periphery of the 053054 epitope, and is therefore is not visible in the crystal structure of the complex (Fig. 5a). R263 is also distant from the epitope of 092096. Nevertheless, to determine the effects of these polymorphisms within or adjacent to the epitopes on the binding of 053054 and 092096 to DBP, single and double mutant DBP variants were generated and analysed for antibody binding by ELISA. All variants retained robust binding to both antibodies (Fig. 5b). We further quantified binding of antibodies to the DBP variants by BLI. Binding affinities showed no difference in equilibrium dissociation constants for the natural DBP variants compared to Sal-I DBP-II (Supplementary Table 4 and Supplementary Figs. 6 and 7). In addition to the most frequent polymorphism, we tested whether the lower-frequency polymorphisms L288F, I374M and T359R affected binding using BLI (Supplementary Table 4 and Supplementary Figs. 6 and 7). I374M occurs most frequently in combination with $\mathrm{N} 372 \mathrm{~K}$, and T359R occurs most frequently with R263S. Introducing these mutations into DBP also had no effect on antibody affinity or binding parameters. These results demonstrate that the polymorphisms within the epitopes do not affect antibody binding.

053054 and 092096 heavy and light chain sequences show limited development from the germline genes. The lack of sequence similarity between the antibodies 053054 and 092096 (Fig. 6a,b) indicates that they did not evolve from a common progenitor. Nevertheless, both antibodies recognize overlapping epitopes in DBP. We analysed the CDRs of the neutralizing antibodies to DBP to determine the degree of deviation of the paratropes from the germline and to what extent the antibodies would need to develop. Nucleotide and amino acid sequences were analysed using NCBI IgBlast and IMGT/V-Quest to identify the germline V, D and J genes (Fig. 6c-f). The analysis showed that 053054 and 092096 heavy- and light-chain sequences are very similar to the germline genes with a minimal number of junction insertions (Supplementary Table 6). A limited number of somatic hypermutations create direct contacts between the antibodies and DBP: 2 (T62 and S110) of 14 contact residues in the 053054 heavy chain; 

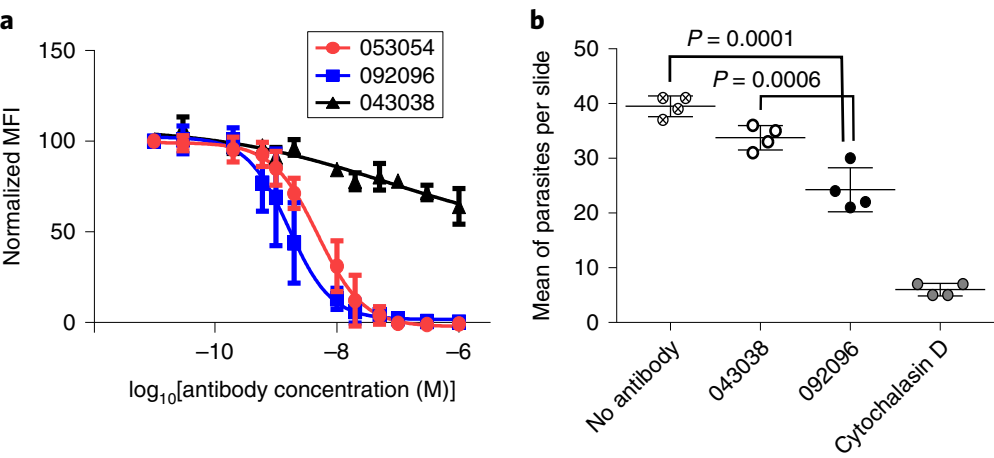

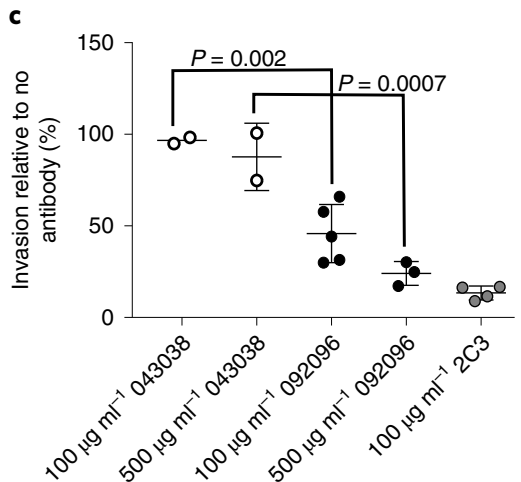

e

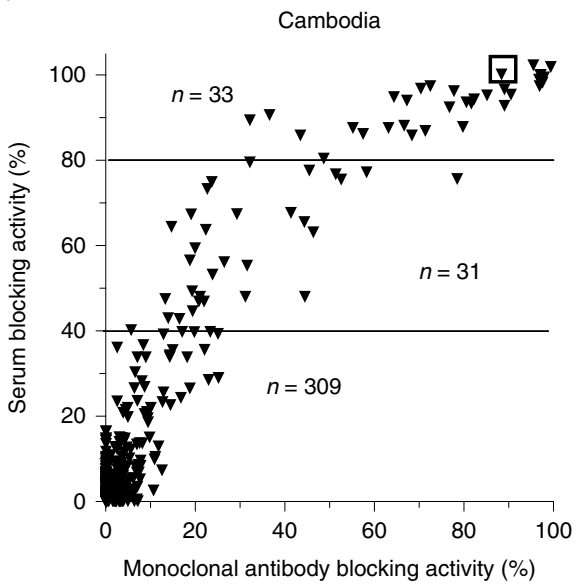

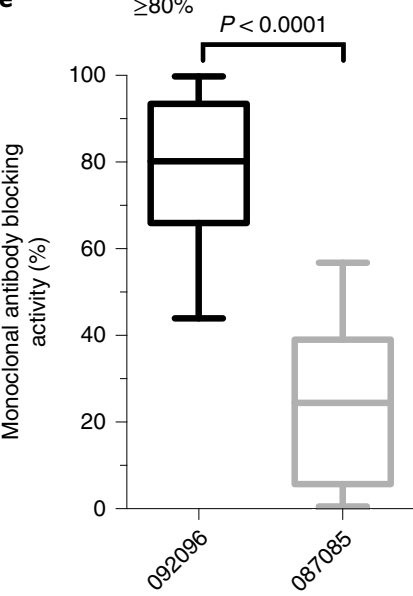

$40-79 \%$

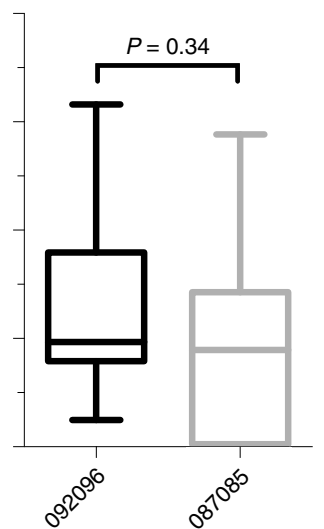

Fig. 3 | Human antibodies block DBP binding to RBCs and P. vivax invasion. a, Inhibition of DBP-II binding to RBCs. The isotype-matched monoclonal antibody 043038 was used as a negative control. Data are mean \pm s.d. of three biological replicates. $\mathrm{MFI}$, mean fluorescence intensity. $\mathbf{b}$, Neutralization data for a Brazilian isolate of $P$. vivax invasion of human reticulocytes by human monoclonal antibody 092096. Individual data points represent the total number of $P$. vivax-infected cells per 20,000 RBCs and are shown as mean \pm s.e.m. for one $P$. vivax isolate performed in four biological replicate cultures in one experiment. Statistical differences between 092096 and no invasion inhibitor control (medium alone) and non-inhibitory monoclonal antibody 043038 were analysed by one-way analysis of variance and Dunnett's test $(P=0.0001$ and 0.0006 , respectively). Cytochalasin $D$, an actin inhibitor, served as a positive control for the inhibition of invasion. c, Neutralization data for Cambodian isolates of $P$. vivax invasion of human reticulocytes by human monoclonal antibody 092096. Each data point represents results from one of five $P$. vivax isolates and the results are presented as mean \pm s.e.m. These data represent results from five separate experiments, each with a different $P$. vivax isolate. All experiments had no antibody control, and four out of five experiments used mouse anti-DARC monoclonal antibody $2 \mathrm{C} 3$ as a positive control and one experiment used heparin as positive control (11\% invasion relative to no antibody, not shown in the figure). The other controls were performed with only some experiments because of the limited number of parasites available for different culture conditions. The isotype-matched monoclonal antibody 043038 serves as an additional negative control. Statistical comparison between noninhibitory antibody $043038(n=2)$ and neutralizing antibody 092096 was performed using one-way analysis of variance and Dunnett's test $(P=0.002$ for $100 \mu \mathrm{g} \mathrm{ml}^{-1}$ antibody concentration, $n=5 ; P=0.0007$ for $500 \mu \mathrm{g} \mathrm{ml}^{-1}$ antibody concentration, $n=3$ ). d, Human monoclonal antibodies compete with epitopes recognized by serum from individuals from Cambodia with high levels of DBP-II-specific blocking activity ( $\geq 80 \%)$. The $y$ axis indicates the level of blocking activity using a DBP-II-DARC binding assay, which measures the overall blocking activity in a serum sample. The $x$ axis is the percentage of blocking activity in the sample for which a given antibody competed. The black box highlighting a data point indicates the serum sample that blocked $100 \%$ of DBP-II binding to DARC $N$ terminus at a dilution of 1:20. e, The box and whisker plots (median blocking activity, 25 and $75 \%$ interquartile ranges and $95 \%$ confidence intervals) show that the degree to which antibodies 092096 and 087085 compete with serum-blocking activity stratifies participants into those with $80-100 \%$ blocking activity ( $n=33$ for both antibodies, $P<0.0001)$ and those with lower blocking activity of $40-79 \%(n=31$ for both antibodies, $P=0.34)$. The sample sizes for the two strata are shown in the scatter plots. Difference in blocking activity was examined using two-tailed Mann-Whitney $U$ test.

4 (E173, H196, T237 and E240) of 15 contact residues in the 053054 light chain; 2 (Y108 and F109) of 20 residues in the 092096 heavy chain; and 3 (R175, P177 and D197) of 14 residues in the 092096 light chain (Supplementary Table 2). The small number of somatic hypermutations reflects that the antibodies underwent limited affinity maturation to achieve broadly neutralizing activity (Supplementary Table 6).

\section{Discussion}

We found that the naturally acquired human antibodies 053054 and 092096 target the DARC-binding site and dimer interface in DBP-II.
The structural and HDX epitope-mapping data demonstrate that the epitope for 053054 spans residues E249, D264A281 and Q356N372 of helices in SD2. This epitope overlaps with the epitope for 092096, which comprises Y219, E249, L270-K289 and Q356-W375. These findings indicate clearly a mechanism for inhibition and neutralization. These human antibodies function by preventing DBP-II binding to the N-terminus of DARC and are thereby neutralizing. Notably, we show that a single human antibody, 092096, which targets the receptor-binding residues and dimer interface of DBP, can neutralize invasion of reticulocytes by multiple $P$. vivax isolates from distinct geographical locations. 

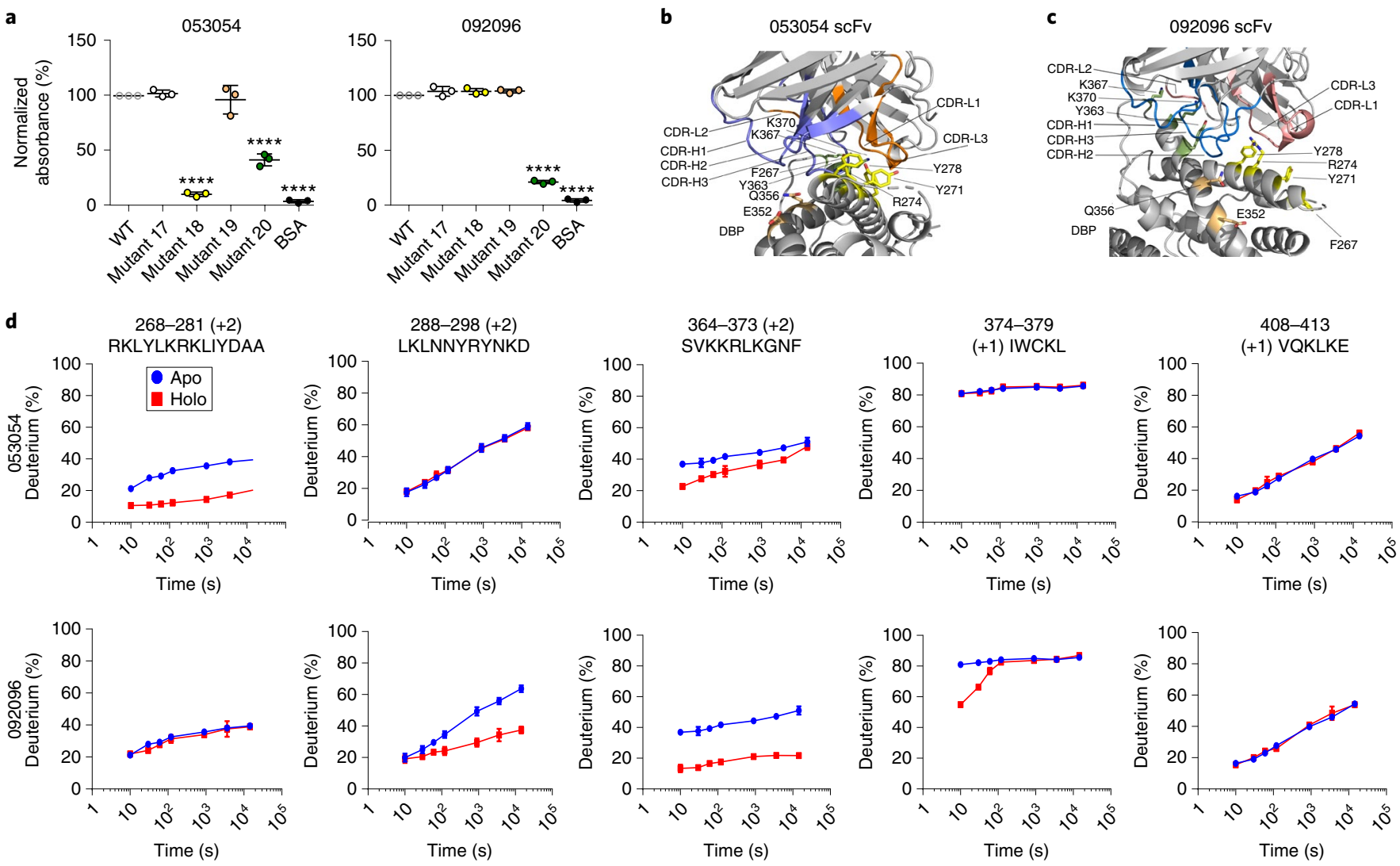

Fig. 4 | Mutant ELISAs and HDX-MS reveal overlapping but distinct binding modes. a, Evaluation of 053054 and 092096 binding to Sal1 DBP-II and four DBP mutants by ELISA. Mutant 17, T257G/D258G/T259S/N260G/F261G/H262S; mutant 18, F267A/Y271A/R274A/Y278A; mutant 19, E352A/Q356A; mutant 20, Y363A/K367A/K370A. WT, wild-type; BSA, bovine serum albumin. Data are mean \pm s.d., calculated from three independent experiments with three technical replicates each; one-way analysis of variance and Dunnett's test ( $\left.{ }^{\star \star \star \star} P<0.0001\right)$. b,c , Position of DBP-II surface mutations tested in a in relation to the individual 053054 (b) and 092096 (c) (see also Supplementary Fig. 2). Grey, DBP-II and antibody; yellow, mutant 18 residues; salmon, mutant 19 residues; green, mutant 20 residues. d, HDX-MS kinetics for five DBP peptides in the presence (red) or absence (blue) of 053054 or 092096. Peptide sequence and charge state is shown (see also Supplementary Fig. 4 for full peptide coverage of DBP). The HDX data were determined in duplicate, and data are presented as mean \pm s.d. For determinations where the deviation is not seen, it is smaller than the size of the data point. The statistical validity of the curves was also assured by making the measurement over seven time points. Given that the purpose of the HDX kinetic plots is to assign binding or no-binding, these assignments were made visually without further statistical analysis.

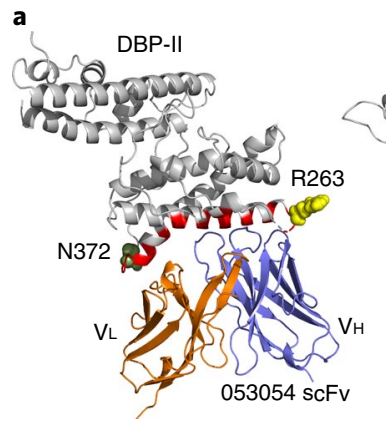

DBP-II

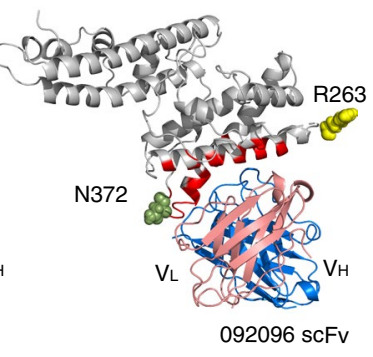

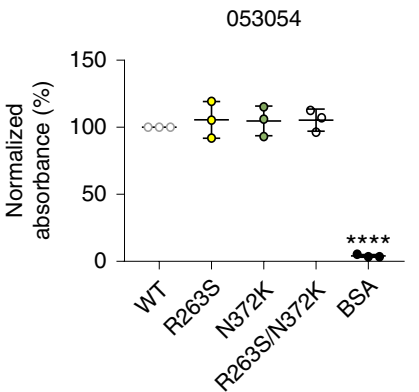

092096

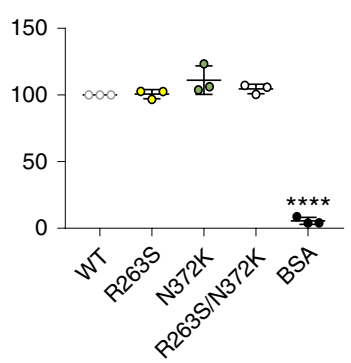

Fig. 5 | Strain-transcending human monoclonal antibodies overcome polymorphisms located within the vicinity of their epitopes. a, Location of polymorphisms R263S and N372K on DBP-II surface shown in DBP-II-053054 and DBP-II-092096 structures. Polymorphic residues 263 and 372 shown in ball representation in yellow and green, respectively. The location of R263 was modelled, as this residue is located in a disordered segment that is not visible in either structure. b, ELISA for 053054 and 092096 against wild-type Sal-I DBP-II and three DBP-II polymorphisms: R263S, N372K and R263S/ N372K. Data are mean \pm s.d., calculated from three independent experiments. Statistical differences between wild-type Sal-1 DBP and the mutants were analysed by one-way analysis of variance and Dunnett's test $\left({ }^{\star \star \star \star} P<0.0001\right)$.

The human antibody epitopes are distinct from broadly neutralizing epitopes of murine monoclonal antibodies ${ }^{19}$, and this diversity of epitopes residing in different subdomains of DBP-II is reminiscent of receptor-binding-site ${ }^{26}$ and stem $^{27}$ epitopes in influenza haemagglutinin. Although 053054 and 092096 do not share a germline lineage, they target overlapping epitopes of DBP 
a

053054 heavy chain 092096_heavy chain

053054_heavy chain 092096_heavy chain b

053054_light chain 092096_light chain

053054_light chain 092096_light chain

c

053054_heavy chain IGHV4-30-4*01(V) IGHD4-23*01(D) $\mathrm{GHJ} 5^{*} 02(\mathrm{~J})$

053054 heavy chain IGHV4-30-4*01(V) IGHD4-23*01(D)

d

053054_light chain IGLV3-21*02(V)
IGLJ2*01(J)

053054_light chain IGLV3-21*02(V)

e

092096 heavy chain
IGHV1-24*01(V) IGHD2-15*01(D)

092096_heavy chain IGHV1-24*01(V) IGHD2-15*01(D)
IGHJ3*02(J)

f

092096 light chain IGLV1-44*01(V) I GLJ2*01(J)

092096_light chain IGLV1-44*01(V)

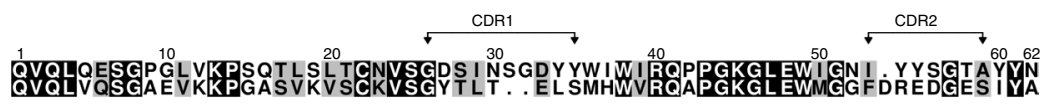

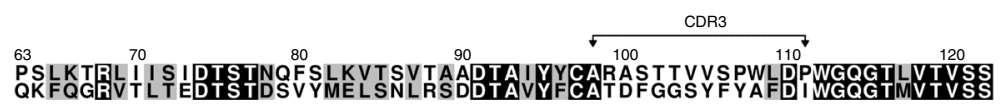

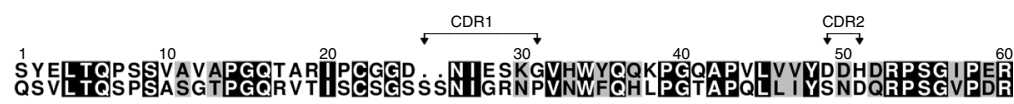

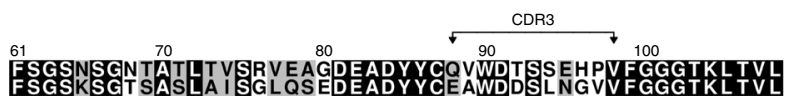

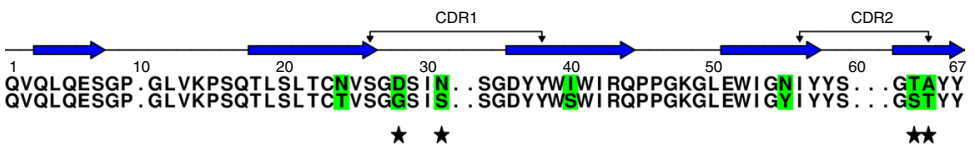
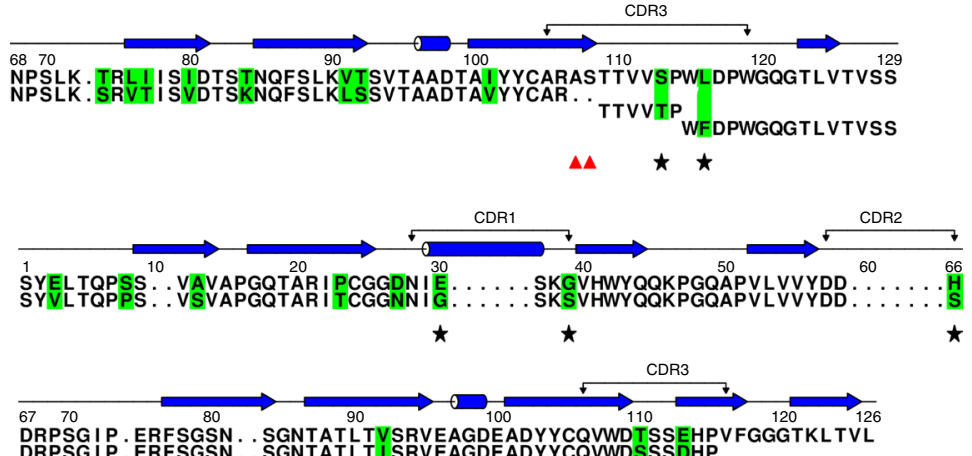

$\star \star$ VFGGGTKLTVL
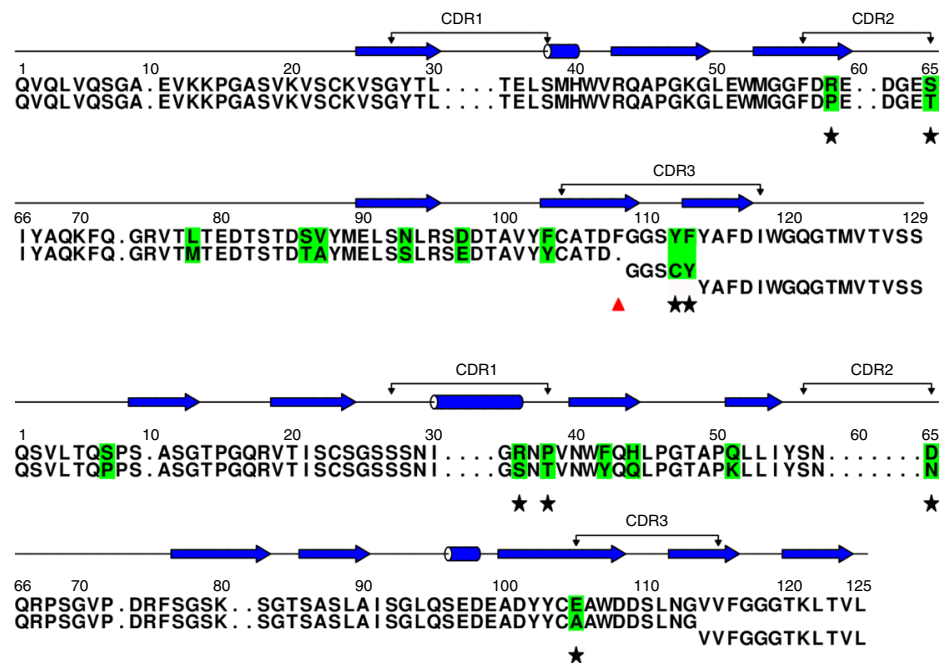

Fig. 6 | Monoclonal antibody maturation. a,b, Sequence identity analysis for 053054 and 092096 by alignment of heavy (a) and light (b) chains. CDRs 1-3 are marked at the top of the alignment. Similar residues are highlighted grey, identical residues are in black. c-f, Amino acid sequence alignment of the variable heavy chain and light chain of the antibodies 053054 (c,d) and 092096 (e,f) against the germline V, D and J gene members with the highest sequence similarity, defined by NCBI IgBlast and IMGT/V-Quest as indicated in each alignment. CDRs 1-3 are shown at the top of the alignment. Amino acid mutations are highlighted green. Non-silent somatic hypermutations in CDRs are indicated by black stars. Red triangles mark insertions in the junction region. The secondary structure elements are indicated by blue arrows for $\beta$-sheets and blue coils for $\alpha$-helices. $\mathbf{g}$, The number of non-silent somatic mutations for CDRs of $\mathrm{VH}_{\mathrm{H}}$ and $\mathrm{VL}$ chains of each antibody as well as junction insertions.

involved in DARC engagement. Our results suggest the development of neutralizing monoclonal antibodies can arise from multiple progenitor B cells with straightforward CDR affinity maturation, resulting in a high potency and breadth of neutralization. The epitopes for broadly neutralizing human antibodies presented in this study are, therefore, an excellent platform to develop vaccines based on DBP-II.

DBP is highly polymorphic, and this variation may confound vaccine development ${ }^{28,29}$. Identification of highly conserved epitopes of neutralizing antibodies will have a profoundly positive 
effect on the development of a DBP-based vaccine. One caveat of the invasion assays presented here is that the tested isolates were not sequenced to identify the diversity of DBP variants. Therefore, we examined the breadth of binding of the antibodies to DBP variants that encompassed polymorphisms within the epitopes. We demonstrated that the most prevalent polymorphisms within or adjacent to the epitopes, as determined by comparative analysis of 599 DBP sequences, were residues N372K, R263S, I374M, L288F and T359R. These polymorphisms, individually and in combination, do not affect antibody binding to DBP-II. This suggests that human neutralizing antibodies have evolved to compensate for polymorphisms and variation in DBP, probably as a result of repeated exposure to $P$. vivax, thereby becoming broadly neutralizing antibodies.

A key challenge for successful vaccination is the need for complex maturation of broadly neutralizing antibodies from their precursors, requiring long developmental pathways. For example, broadly neutralizing antibodies that target HIV must undergo substantial development, including multiple rounds of affinity maturation, to result in CDRs capable of neutralization breadth, involving extensive lengthening of the CDRs ${ }^{30}$. This has had a fundamental impact on the immunization scheme necessary to generate suitable broadly neutralizing antibodies for HIV. By contrast, the number of somatic hypermutations seen here reflects that 053054 and 092096 underwent limited affinity maturation for broadly neutralizing activity. This suggests that immunization with DBP-based vaccines is likely to lead to production of neutralizing antibodies.

The findings from this study of DBP will inform neutralization of and vaccine development for an important family of parasite invasion ligands. DBP is a member of the erythrocyte-binding-like (EBL) family of Plasmodium red cell invasion proteins that includes EBA-175 $5^{31-37}$, EBA-140 ${ }^{38-44}$, EBA-181 ${ }^{43,45-47}$ and EBL- $1^{48}$. EBL family members have either one or two tandem DBL domains, which are used by Plasmodium parasites for receptor binding and attachment to host erythrocytes. This study has established that a single monoclonal antibody that targets the receptor-binding residues of DBP can neutralize $P$. vivax parasites, and underscores the importance of targeting the functional receptor-binding residues of other EBL ligands. In addition, DBP and EBA-175 undergo receptor-induced dimerization required for invasion ${ }^{17,18,34,35}$. The antibodies 053054 and 092096 also block the dimer interface of DBP, suggesting that antibody disruption of oligomeric interfaces in antigens could be a general mechanism for neutralization. Defining the full complement of mechanisms for neutralizing DBL domains using antibodies will aid in the direct design of highly efficient immunogens against malaria. Although additional receptor-ligand interactions have been identified with proposed roles in $P$. vivax malaria ${ }^{49,50}$, a $P$. vivax vaccine is likely to include $\mathrm{DBP}$, given the central role of the DBP-DARC interaction in infection.

In conclusion, we studied two human neutralizing monoclonal antibodies, 053054 and 092096, that target $P$. vivax DBP isolated from an individual with naturally acquired immunity to $P$. vivax and with high levels of blocking antibodies to DBP. These two monoclonal antibodies arose from different clonal groups, bind overlapping epitopes in DBP, and compete with antibodies found extensively in multiple patients with blocking antibody activity to DBP. We characterized their interaction with DBP, confirmed their high neutralizing potential, structurally defined their epitopes and proposed a mechanism for neutralization. This work extends our knowledge of the function of parasitic ligands and methods to leverage the immune response for neutralization. These naturally acquired human antibodies appear to have a short pathway for development with broadly neutralizing capacity. Monoclonal antibodies to DBP-II following immunization of mice recognized a distinct set of epitopes that do not target the receptor-binding domain. Therefore, current vaccination protocols may not favour targeting the receptor-binding domain and dimer interface of
DBP to generate antibodies capable of blocking parasite invasion. Furthermore, while naturally acquired immunity can result in the desired neutralizing antibodies described here, it requires repeated exposure to the parasite and develops over the lifetime of a patient. The structural definition of conformational epitopes provides a solid foundation for focusing the immune response to the neutralizing human antibody epitopes through immunogen design and structural vaccinology. This work informs the design of immunogens that primarily elicit antibodies to the neutralizing epitopes identified here and to avoid or reduce targeting immunodominant but non-protective epitopes within DBP. Improved immunogens will aid in the design of successful and efficient DBP-based vaccines for malaria.

\section{Methods}

Cell staining and sorting of antigen-specific memory B cells. Blood samples were obtained from donors residing in P. vivax-endemic areas ${ }^{2,51-53}$. in Cambodia, Brazil and Papua New Guinea. Samples were screened for blocking antibodies to DBP-II (see below). A subset of Cambodian and Brazilian adults with high levels of blocking antibodies to DBP-II ( $>80 \%$ binding-inhibitory activity at a titre $1: 10$ or higher) donated up to $200 \mathrm{ml}$ of peripheral venous blood. Peripheral blood mononuclear cells (PBMCs) were prepared from the venous blood and cryopreserved. Institutional review boards from the US National Institutes of Health (NIAID protocol \#08-N094, ClinicalTrials.gov NCT00663546), Cambodian Ministry of Health, University Hospital of the University of Sao Paulo (1025/10), National Human Research Ethics Committee of the Ministry of Health of Brazil (551/2010), Medical Research Advisory Counsel of Papua New Guinea (PNGIMR No 1409, PNG MRAC No. 1400 and UH IRB No. 04-14-19) and University Hospitals of Cleveland Medical Center approved the protocols. Written informed consent was obtained from all study participants or their parents or guardians.

Single cells were identified and sorted according to previously described techniques $^{54}$ from cryopreserved PBMCs without activation. B cells were enriched from cryopreserved PBMCs using immunomagnetic positive selection with anti-CD19 magnetic MACS beads (Miltenyi Biotec). Cells were washed at least twice with $5 \mathrm{ml}$ FACS buffer with $3 \mathrm{mM}$ EDTA and adjusted to a cell density of $1 \times 10^{6}-2 \times 10^{6}$ cells per ml. Cells were stained with mouse anti-human CD20 (PE-Cy5.5, Invitrogen) and anti-human IgG (PE-Cy7 clone G18-145; Becton Dickinson) along with DBP-II- or TTCF-prepared tetramers using streptavidin coupled with allophycocyanin (Becton Dickinson) and SYTOX Green Dead Cell Stain (Invitrogen) to gate out dead cells. Stained CD19 ${ }^{+}$cells were sorted on a BD FACSAria II based on size and complexity and individual DBP-II- or TTCFspecific $\mathrm{CD} 20^{+}, \mathrm{IgG}^{+}$memory B cells were sorted as single cells directly into $4 \mu \mathrm{l}$ mRNA extraction buffer on a cooled 96-well metal block. After cells were collected, plates were frozen immediately on dry ice and stored at $-80^{\circ} \mathrm{C}$ until further processing.

Complementary DNA synthesis. The 96-well plates with single cells were thawed on ice; $7 \mu \mathrm{l}$ of solution containing $300 \mathrm{ng}$ random hexamers (Qiagen Operon), $12 \mathrm{U}$ Rnasin (Promega) and 0.9\% NP-40 (Thermo Scientific Pierce) was added to each well. After thorough pipetting and rinsing, wells were capped, centrifuged at $4{ }^{\circ} \mathrm{C}$, heated to $68^{\circ} \mathrm{C}$ in a thermal cycler for $5 \mathrm{~min}$ and placed on ice for at least $1 \mathrm{~min}$. Reverse transcription was performed with the addition of $7 \mu$ l containing $3.6 \mu \mathrm{l}$ $5 \times$ reverse transcriptase buffer, 10 U RNAsin (Promega), 62 U Superscript III RT (Invitrogen), $0.62 \mu \mathrm{l} \mathrm{dNTPs} \mathrm{(25} \mathrm{mM} \mathrm{each;} \mathrm{Omega} \mathrm{Bio-Tek)} \mathrm{and} 1.25 \mu \mathrm{l} 0.1 \mathrm{M}$ DTT (Sigma). All wells were capped and the plate was placed in a cold rack and vortexed for $10 \mathrm{~s}$ before centrifugation at $300 \mathrm{~g}$. Thermal cycler conditions for reverse transcription were as follows: $42^{\circ} \mathrm{C}$ for $5 \mathrm{~min}, 25^{\circ} \mathrm{C}$ for $10 \mathrm{~min}, 50^{\circ} \mathrm{C}$ for $60 \mathrm{~min}$, $94^{\circ} \mathrm{C}$ for $5 \mathrm{~min}$ and $4^{\circ} \mathrm{C}$ hold. When completed, $10 \mu$ of nuclease-free PCR water was added to each well.

Immunoglobulin gene amplification. Immediately following complementary DNA synthesis, IgG genes (Igg) were amplified in a total of $20 \mu \mathrm{l}$ per well for the first round of nested PCR for IgG heavy chain (Iggh), IgG kappa (Iggא) and IgG lambda (Igg $\lambda$ ), utilizing primers (Supplementary Table 1 ) as previously described ${ }^{55}$ In brief, a master mix was prepared, consisting of $15.58 \mu \mathrm{l}$ water, $2 \mu \mathrm{l} 10 \times$ HotStar PCR buffer (Qiagen), $0.065 \mu \mathrm{l} \mathrm{5'}$ primer mix, $0.065 \mu \mathrm{l} \mathrm{3} 3^{\prime}$ primer, $0.2 \mu \mathrm{l} \mathrm{dNTP}$ solution and $0.09 \mu \mathrm{l} \mathrm{HotStarTaq}$ per well, to which $2 \mu \mathrm{l} \mathrm{cDNA}$ from individual sorted B cells were added and Igg was amplified under the following conditions: thermal cycle PCR at $94^{\circ} \mathrm{C}$ for $15 \mathrm{~min} ; 50$ cycles at $94^{\circ} \mathrm{C}$ for $30 \mathrm{~s}, 58^{\circ} \mathrm{C}$ (Iggh and Iggא) or $60^{\circ} \mathrm{C}(\operatorname{Igg} \lambda)$ for $30 \mathrm{~s}, 72^{\circ} \mathrm{C}$ for $55 \mathrm{~s}$; then one cycle at $72^{\circ} \mathrm{C}$ for $10 \mathrm{~min}$. A second round of nested PCR for Iggh, Iggא and Igg $\lambda$ utilized $2 \mu$ l of the first-round PCR product with second-round primers ${ }^{55}$ and the same master mix protocol, with the following conditions: thermal cycle PCR at $94^{\circ} \mathrm{C}$ for $15 \mathrm{~min} ; 50$ cycles at $94^{\circ} \mathrm{C}$ for $30 \mathrm{~s}, 58^{\circ} \mathrm{C}$ (Iggh and Iggא) or $60^{\circ} \mathrm{C}(\operatorname{Igg} \lambda)$ for $30 \mathrm{~s}, 72^{\circ} \mathrm{C}$ for $45 \mathrm{~s}$; then one cycle at $72^{\circ} \mathrm{C}$ for $10 \mathrm{~min}$. The PCR product generated was purified and sequenced, with $\mathrm{V}(\mathrm{D}) \mathrm{J}$ genes determined using IMGT/V-Quest ${ }^{56}$. 
Specific V(D)J region amplification and cloning. Primers specific for $\mathrm{V}$ and J regions, with restriction enzyme sites, were used to amplify the first-round PCR product to generate a fragment for cloning, based on previously described primers $^{55}$. PCR product was purified, digested using restriction enzymes and cloned into Iggh, Iggא or Igg $\lambda$ expression vectors and chemically transformed into $5 \mu \mathrm{l}$ aliquots of TOP10 Escherichia coli cells (Thermo Fisher Scientific). Successful transformants were screened by PCR amplification using a vector-specific primer paired with an insert-specific primer, sequenced and compared to the secondround PCR product sequence.

Definition of clonal groups. Clonal groups were based on heavy chain nucleotide sequences. Any PCR product with $>0.8 \%$ nucleotide sequences with a Phred score $<20$ was excluded. From PCR-amplified sequences, we determined heavy chain alleles using IMGT/V-Quest (http://www.imgt.org). A clonal group was defined by antibody $\mathrm{V}$ heavy chain sequences with the same inferred $\mathrm{VH}$ and $\mathrm{JH}$ germline sequences, identical CDR3 length, and the same or very similar CDR3 sequences, that is, $>72 \%$ similarity of each CDR3 amino acid sequence. Clonal grouping was determined using Sequence Manipulation Suite: Ident and Sim ${ }^{57}$ using antibody-specific clusters as previously defined ${ }^{58}$.

Monoclonal antibody expression and purification. Two plasmids that included coding sequences for full-length IgG1 heavy and light chains were transfected into HEK293-H cells using polyethyleneimine (PEI). Five hundred micrograms PEI was incubated for $25 \mathrm{~min}$ at room temperature with $250 \mu \mathrm{g}$ of each plasmid and then added to the HEK293-H at a density of $1 \times 10^{6}$ cells per $\mathrm{ml}$ in a total volume of $500 \mathrm{ml}$ (refs. ${ }^{54,55}$ ). Transfected cells were adapted for growth in Freestyle 293 serum-free expression medium (Gibco, Thermo Fisher Scientific) under suspension conditions of $37^{\circ} \mathrm{C}$ and $8.5 \% \mathrm{CO}_{2}$. Cells were centrifuged $96 \mathrm{~h}$ after transfection and culture medium was collected, filtered through a $0.22 \mu \mathrm{m}$ filter, and supernatants were concentrated 20 times using a $50 \mathrm{kDa}$ cut-off Vivaflow 50 System (Vivasciences). One volume of IgG binding buffer (Thermo Scientific Pierce) was added and IgG was purified on a Protein A HP HiTrap column (GE Healthcare) eluted with IgG elution buffer (Thermo Scientific Pierce) and neutralized with $1 \mathrm{M}$ Tris $\mathrm{pH}$ 9.0. Proteins were concentrated and buffer exchanged with PBS using Amicon Ultra4 $10 \mathrm{kDa}$. Protein concentrations were determined on a Nanodrop (Thermo Fisher Scientific) and sample purity was analysed by SDS-PAGE.

Protein expression and purification. Sal-1 DBP-II wild-type and DBP-II mutants were prepared as described ${ }^{17-19}$. scFvs were produced by expression and refolding from $E$. coli. The light chain variable region was linked to the heavy chain variable region using a (GGGGS) ${ }_{3}$ linker, cloned into the $\mathrm{pET} 28(\mathrm{a}+)$ expression vector using restriction sites $\mathrm{NcoI}$ and XhoI, and expressed in E. coli. Inclusion bodies were solubilized in $6 \mathrm{M}$ guanidinium hydrochloride overnight at $4{ }^{\circ} \mathrm{C}$ and refolded with $400 \mathrm{mM}$ L-arginine, $2 \mathrm{mM}$ reduced glutathione and $0.2 \mathrm{mM}$ oxidized glutathione in $50 \mathrm{mM}$ Tris, $\mathrm{pH}$ 8.0, $10 \mathrm{mM}$ EDTA and $0.1 \mathrm{mM} \mathrm{PMSF}$ at $4{ }^{\circ} \mathrm{C}$ for $24 \mathrm{~h}$. Refolded scFv was concentrated in Amicon Stirred Cells using Biomax $10 \mathrm{kDa}$ Ultracentrifugation Discs and purified by size-exclusion chromatography on GF200 (GE Healthcare) into $10 \mathrm{mM}$ HEPES pH 7.4, $100 \mathrm{mM} \mathrm{NaCl}$

Protein crystallization and data collection. Purified DBP-II and scFvs of 053054 or 092096 were mixed in a 1.2:1 (antigen:antibody) molar ratio and incubated at room temperature for $30 \mathrm{~min}$. Complexes were purified by size-exclusion chromatography (GF200, GE Healthcare) in $10 \mathrm{mM}$ HEPES pH 7.4, $100 \mathrm{mM} \mathrm{NaCl}$. Crystals were grown by hanging-drop vapour diffusion by mixing $1 \mu \mathrm{l}$ of complex at $7 \mathrm{mg} \mathrm{ml}^{-1}$ for DBP-II-053054 or $10 \mathrm{mg} \mathrm{ml}^{-1}$ for DBP-II-092096 with $2 \mu \mathrm{l}$ of reservoir $(0.2 \mathrm{M}$ ammonium sulfate, $0.1 \mathrm{M}$ sodium citrate $\mathrm{pH} 5.6,25 \% \mathrm{w} / \mathrm{v}$ PEG 4,000 for DBP-II-053054 or 12\% w/v PEG 6,000, 0.1 M MES, pH 6.0 for DBPII-092096). Crystals were flash frozen with $30 \%$ ethylene glycol as cryoprotectant in liquid nitrogen. Data for DBP-II-053054 were collected at the beamline 4.2.2 of the Advanced Light Source. Data for DBP-II-092096 were collected at the beamline 23-ID-D (GM/CA) of the Advanced Photon Source. Both datasets were processed with $\mathrm{XDS}^{59}$.

Structure solution and analysis. The DBP-II-053054 and DBP-II-092096 structures were solved by molecular replacement in PHASER ${ }^{60}$ by using PDB $4 \mathrm{NUV}$ and $\mathrm{scF}$ V domains modelled by the PIGS server ${ }^{61}$ as search models. Refinement was performed using PHENIX ${ }^{62}$ and COOT ${ }^{63}$. Summary of diffraction data and refinement statistics are given in Supplementary Table 1 . The quality of the models was assessed using the MolProbity server ${ }^{64}$. All structures have good Ramachandran statistics with no outliers. In total, $94.89 \%$ and $5.11 \%$ of residues for DBP-II-053054 or $93.80 \%$ and $6.20 \%$ of residues for DBP-II-092096 were in favoured and allowed regions of Ramachandran plot, respectively. Interaction interfaces were determined using PDBePISA ${ }^{65}$. Software used in the project was installed and configured by SBGrid ${ }^{66}$.

HDX-MS. Before HDX-footprinting experiments, $10 \mu \mathrm{M}$ DBP-II was incubated with $12 \mu \mathrm{M}$ of monoclonal antibody in PBS for $30 \mathrm{~min}$ at $25^{\circ} \mathrm{C}$. The apo-state sample was $10 \mu \mathrm{M}$ DBP in PBS. Continuous HDX on apo- and holo-state samples was performed after diluting into $\mathrm{D}_{2} \mathrm{O}$ buffer at the ratio of $1: 5$ and incubated for $10,30,60,120,900,3,600$ and $14,400 \mathrm{~s}$, as described previously ${ }^{19}$, followed by online pepsin digestion and reversed-phase HPLC separation. Peptides were analysed by a Thermo LTQ-FT mass spectrometer (Thermo Scientific). Deuterium uptakes of various time points were analysed using HDX Workbench (Scripps).

ELISA. ELISAs were performed as previously described ${ }^{36}$. In brief, BSA, Sall DBP-II, and DBP-II mutants were coated on plates overnight at $4{ }^{\circ} \mathrm{C}$, washed with PBS-Tween-20, and blocked with $2 \%$ BSA in PBS-Tween-20 for $1 \mathrm{~h}$ at room temperature. The plates were washed with PBS-Tween-20 and then incubated with each antibody ( 053054 or 092096 ) at a concentration of $250 \mathrm{ng} \mathrm{ml}^{-1}$ for $1 \mathrm{~h}$ at room temperature. The plates were washed with PBS-Tween-20 and incubated with an anti-human antibody conjugated to HRP for $30 \mathrm{~min}$ at room temperature. After a final wash step with PBS-Tween-20 ELISA, substrate TMB was added to the plates The reaction was quenched by addition of $0.2 \mathrm{M}$ sulfuric acid, and the absorbance at $450 \mathrm{~nm}$ was measured using a POLARstar Omega (BMG Labtech) plate reader.

Biotinylation of BirA-tagged Sal-1 DBP-II. BirA-tagged Sal-1 DBP-II was buffer exchanged into PBS. Then, $50 \mu \mathrm{l}$ of BiomixA (Avidity), $50 \mu \mathrm{l}$ of BiomixB (Avidity) and $10 \mu \mathrm{l}$ of $5 \mathrm{mM}$ D-biotin (Avidity) were added to the protein along with BirA ligase, followed by overnight incubation at $4{ }^{\circ} \mathrm{C}$. The biotinylation was confirmed by western blot using Streptavidin-HRP conjugate (Thermo Scientific). Before use, the reaction mix was buffer exchanged into PBS.

BLI assays. The binding affinity of purified Sal1 DBP-II protein with human monoclonal antibodies was monitored by BLI on an Octet-Red96 device (Pall ForteBio) using streptavidin biosensors (ForteBio). The biotinylated DBP-II was loaded onto biosensors until saturation, typically $1 \mu \mathrm{M}$ for $15 \mathrm{~min}$, in $10 \mathrm{mM}$ HEPES (pH 7.4), $150 \mathrm{mM} \mathrm{NaCl}, 3 \mathrm{mM}$ EDTA and $0.005 \%$ P20 surfactant with $3 \% \mathrm{BSA}$. The scFvs (analyte) were applied at twofold serial dilution concentrations ( $100 \mathrm{nM}$ to $1.675 \mathrm{nM})$. Association and dissociation were measured at $25^{\circ} \mathrm{C}$ for all antibodies. The real-time data were analysed using Biaevaluation 4.1 (GE Healthcare). Steadystate equilibrium concentration curves were fitted using a 1:1 binding model.

FACS. Biotinylated purified DBP-II protein at $10 \mathrm{nM}$ was incubated with ten different concentrations of 053054 or 092096 (at intervals of $0.03 \mathrm{nM}$ to $1 \mu \mathrm{M}$ ) for $1 \mathrm{~h}$ at room temperature. Red blood cells were added and incubated for an additional $1 \mathrm{~h}$ at room temperature. To detect bound DBP, Alexa Fluor 488streptavidin conjugate (Fisher) was added to the mix and incubated at room temperature for $1 \mathrm{~h}$ followed by washing twice with PBS. Labelling with fluorescein isothiocyanate (FITC) was measured by flow cytometry. An $\mathrm{IC}_{50}$ value was calculated in GraphPad Prism from three independent biological replicates. The $\mathrm{IC}_{50}$ curve for the isotype control antibody 043038 was plotted as a negative control.

P. vivax invasion assay. $P$. vivax ex vivo assays were performed independently with clinical isolates from Brazil and Cambodia. Cryopreserved $P$. vivax-infected erythrocytes (iRBC) obtained from Brazilian donors with acute $P$. vivax malaria were thawed, enriched using Percoll density gradients, immediately re-suspended in $100 \mu$ l Iscove's modified Dulbecco's medium (IMDM) plus $10 \%$ human $\mathrm{AB}$ serum with GlutaMax $(1: 100)$ to a haematocrit of $6 \%$ and cultured with a gas mix of $5 \% \mathrm{O}_{2}, 5 \% \mathrm{CO}_{2}$ and $90 \% \mathrm{~N}_{2}$ at $37^{\circ} \mathrm{C}$ until established. The cultures were performed in quadruplicate from a single isolate of $P$. vivax and incubated with 092096 or isotype control comprising anti-TT monoclonal antibody 043038 at a concentration of $100 \mu \mathrm{g} \mathrm{ml}^{-1}$ and examined for parasite viability and maturation three times: after $20 \mathrm{~h}$ of initial incubation (initial growth from ring stages into early trophozoites, since trophozoites and schizont stages of the parasites do not survive cryopreservation); after additional incubation for $38 \mathrm{~h}$, at which time most of the parasite have matured to schizont stages prior to merozoite release; and parasite cultures which were supplemented with fresh blood cells enriched for reticulocytes to $\sim 2.5-3.0 \%$ at a ratio of $1: 1$ and additionally incubated for $24 \mathrm{~h}$ to allow for new erythrocyte invasion. The cultures were performed in duplicate and incubated with 092096 or isotype control comprising anti-TT monoclonal antibody 043038 at a concentration of $100 \mu \mathrm{g} \mathrm{ml}^{-1}$ and examined for parasite viability and maturation three times: after $20 \mathrm{~h}$ of initial incubation (initial growth from ring stages into early trophozoites, since trophozoites and schizont stages of the parasites do not survive cryopreservation), after additional incubation for $38 \mathrm{~h}$, at which time most of the parasite have matured to schizont stages prior to merozoite release, then parasite cultures were supplemented with fresh blood cells enriched for reticulocytes to $\sim 2.5-3.0 \%$ at a ratio of $1: 1$ and additional incubation for $24 \mathrm{~h}$ to allow for new erythrocyte invasion. For examination, two smear slides were prepared for each culture well and stained with Giemsa stain. Rings and early trophozoites per 20,000 RBC were counted with blinding three times. Cytochalasin $\mathrm{D}\left(5 \mu \mathrm{g} \mathrm{ml}^{-1}\right)$, a cell-permeable fungal toxin that inhibits merozoite invasion of erythrocytes, was used as a positive invasion inhibitor control.

For the assay with Cambodian isolates, cryopreserved iRBCs obtained from Cambodian patients with acute $P$. vivax malaria were thawed and cultured in IMDM (Gibco) supplemented with 0.5\% Albumax II (Gibco), 2.5\% heat-inactivated human serum, $25 \mathrm{mM}$ HEPES (Gibco), $20 \mu \mathrm{g} \mathrm{ml}^{-1}$ gentamicin (Sigma) and $0.2 \mathrm{mM}$ hypoxanthine (C-C Pro) for $\sim 24$ or $\sim 48 \mathrm{~h}$ until a majority of schizont-stage parasites 
were noted. The schizont-infected erythrocytes were enriched using Percoll-KCl as described $^{67}$, then mixed at a 1:1 ratio of erythrocytes with reticulocytes enriched from cord blood and labelled with CellTrace Far Red dye. The cultures were incubated for $\sim 8 \mathrm{~h}$ in a final volume of $100 \mu \mathrm{l}$ in 96 -well plates or $20 \mu \mathrm{l}$ in 384 -well plates, in the presence of the human monoclonal antibodies 092096 or anti-TT monoclonal antibody 043038 , while a positive invasion inhibitor control used the mouse antiDARC monoclonal antibody $2 \mathrm{C}^{68}$ at $100 \mu \mathrm{g} \mathrm{ml}^{-1}$. Cells were stained with the DNA stain Hoechst 33342 after invasion and examined by flow cytometry. Reticulocytes showing positive Hoechst 33342 and far red staining were scored as new invasion events. Invasion of reticulocytes ranged from $0.19 \%$ to $6.52 \%$ (media alone) for the six experiments, each of which corresponded to one of six $P$. vivax isolates.

DBP-II patient population ELISAs and binding inhibition of DBP-II to DARC fusion protein. To assess antibody blocking activity, plasma from $P$. vivax-exposed individuals was incubated with DBP-II at specified concentrations. We then measured levels of binding inhibition of DBP-II to a fusion protein containing amino acids 1-60 from the DARC N-terminal region fused to the Fc region of human IgG (nDARCIg) ${ }^{5,69}$. Pooled plasma samples from Papua New Guinea or Brazil with high blocking activity served as positive controls. Diluent alone or pooled samples from North American donors not exposed to malaria were used for negative controls. Percent inhibition was calculated as (1 - (optical density (OD) of test sample/OD of negative control) $\times 100$ ).

Competition experiments. For assays measuring competition between monoclonal antibodies and naturally acquired antibodies, plates were coated with monoclonal antibodies at $0.5 \mu \mathrm{g} \mathrm{ml}^{-1}$ per well and incubated overnight at $4{ }^{\circ} \mathrm{C}$, then washed with PBS-Tween-20 and blocked with 3\% BSA in PBS-Tween-20 for $2 \mathrm{~h}$ at room temperature. Serum from naturally immune donors, at a dilution of $1 / 50$, was pre-incubated with biotinylated DBP-II SalI $\left(20 \mathrm{ng} \mathrm{ml}^{-1}\right)$ for $20 \mathrm{~min}$ at room temperature. The serum mixture was added to plates and incubated $1 \mathrm{~h}$ at room temperature. Detection of DBP-II was achieved using High Sensitivity Streptavidin-HRP (Thermo Fisher Scientific) as described for competition assays between individual monoclonal antibodies.

DBP epitope conservation analysis. A total of 599 sequences representing global variation in DBP-II ${ }^{70}$ was obtained from GenBank (accession codes XM_001608337.1, DQ156519, AF289480-AF289483, AF289635-AF289653, AF291096, AY970837AY970925, AF469515-AF469602, U50575-U50590, DQ156513, DQ156515, DQ156522-DQ156523, AF215737-AF215738, AF220657, AF220659-AF220667, FJ491142-FJ491241, EF219451, EF368159-EF368180, EF379127-EF379132, EF379134, GU143914-GU144013, DQ156520, EU812839-EU812960 and EU860428-EU860438), aligned using ClustalW ${ }^{71}$ and inspected in JalView ${ }^{72}$. All 599 sequences contained complete coverage for the 053054 and 092096 epitopes.

Antibody evolution analysis. To compare heavy and light chains of 053054 and 092096 with germlines we used IMGT/V-Quest, an integrated software program for immunoglobulin and $\mathrm{T}$ cell receptor $\mathrm{V}-\mathrm{J}$ and $\mathrm{V}-\mathrm{D}-\mathrm{J}$ rearrangement analysis ${ }^{73}$.

Statistical analyses. Analysis of ELISA data was performed using Prism v.6.03 (GraphPad Software).

Reporting Summary. Further information on research design is available in the Nature Research Reporting Summary linked to this article.

\section{Data availability}

Atomic coordinates and structural factors have been deposited in the Protein Data Bank with accession codes $6 \mathrm{OAN}$ and $6 \mathrm{OAO}$.

Received: 29 October 2018; Accepted: 16 April 2019; Published online: 27 May 2019

\section{References}

1. Guerra, C. A. et al. The international limits and population at risk of Plasmodium vivax transmission in 2009. PLoS Negl. Trop. Dis. 4, e774 (2010).

2. King, C. L. et al. Naturally acquired Duffy-binding protein-specific binding inhibitory antibodies confer protection from blood-stage Plasmodium vivax infection. Proc. Natl Acad. Sci. USA 105, 8363-8368 (2008).

3. Chootong, P. et al. Mapping epitopes of the Plasmodium vivax Duffy binding protein with naturally acquired inhibitory antibodies. Infect. Immun. 78, 1089-1095 (2010).

4. Cole-Tobian, J. L. et al. Age-acquired immunity to a Plasmodium vivax invasion ligand, the Duffy binding protein. J. Infect. Dis. 186, 531-539 (2002).

5. Grimberg, B. T. et al. Plasmodium vivax invasion of human erythrocytes inhibited by antibodies directed against the Duffy binding protein. PLoS Med. 4, e337 (2007).

6. Michon, P., Fraser, T. \& Adams, J. H. Naturally acquired and vaccine-elicited antibodies block erythrocyte cytoadherence of the Plasmodium vivax Duffy binding protein. Infect. Immun. 68, 3164-3171 (2000).
7. McGregor, I. A. The passive transfer of human malarial immunity. Am. J. Trop. Med. Hyg. 13(suppl.), 237-239 (1964).

8. Miller, L. H., Mason, S. J., Dvorak, J. A., McGinniss, M. H. \& Rothman, I. K. Erythrocyte receptors for (Plasmodium knowlesi) malaria: Duffy blood group determinants. Science 189, 561-563 (1975).

9. Miller, L. H., Mason, S. J., Clyde, D. F. \& McGinniss, M. H. The resistance factor to Plasmodium vivax in blacks. The Duffy-blood-group genotype, FyFy. N. Engl. J. Med. 295, 302-304 (1976).

10. Wertheimer, S. P. \& Barnwell, J. W. Plasmodium vivax interaction with the human Duffy blood group glycoprotein: identification of a parasite receptor-like protein. Exp. Parasitol. 69, 340-350 (1989).

11. Adams, J. H. et al. The Duffy receptor family of Plasmodium knowlesi is located within the micronemes of invasive malaria merozoites. Cell 63, 141-153 (1990).

12. Adams, J. H. et al. A family of erythrocyte binding proteins of malaria parasites. Proc. Natl Acad. Sci. USA 89, 7085-7089 (1992).

13. Chitnis, C. E. \& Miller, L. H. Identification of the erythrocyte binding domains of Plasmodium vivax and Plasmodium knowlesi proteins involved in erythrocyte invasion. J. Exp. Med. 180, 497-506 (1994).

14. Chitnis, C. E., Chaudhuri, A., Horuk, R., Pogo, A. O. \& Miller, L. H. The domain on the Duffy blood group antigen for binding Plasmodium vivax and P. knowlesi malarial parasites to erythrocytes. J. Exp. Med. 184, 1531-1536 (1996).

15. Ranjan, A. \& Chitnis, C. E. Mapping regions containing binding residues within functional domains of Plasmodium vivax and Plasmodium knowlesi erythrocytebinding proteins. Proc. Natl Acad. Sci. USA 96, 14067-14072 (1999).

16. VanBuskirk, K. M., Sevova, E. \& Adams, J. H. Conserved residues in the Plasmodium vivax Duffy-binding protein ligand domain are critical for erythrocyte receptor recognition. Proc. Natl Acad. Sci. USA 101, 15754-15759 (2004).

17. Batchelor, J. D., Zahm, J. A. \& Tolia, N. H. Dimerization of Plasmodium vivax DBP is induced upon receptor binding and drives recognition of DARC. Nat. Struct. Mol. Biol. 18, 908-914 (2011).

18. Batchelor, J. D. et al. Red blood cell invasion by Plasmodium vivax: structural basis for DBP engagement of DARC. PLoS Pathogens 10, e1003869 (2014).

19. Chen, E. et al. Broadly neutralizing epitopes in the Plasmodium vivax vaccine candidate Duffy Binding Protein. Proc. Natl Acad. Sci. USA 113, 6277-6282 (2016)

20. Payne, R. O. et al. Human vaccination against Plasmodium vivax Duffy-binding protein induces strain-transcending antibodies. JCI Insight 2, e93683 (2017).

21. Singh, K. et al. Malaria vaccine candidate based on Duffy-binding protein elicits strain transcending functional antibodies in a Phase I trial. NPJ Vaccin. 3, 48 (2018).

22. Cole-Tobian, J. L. et al. Strain-specific Duffy binding protein antibodies correlate with protection against infection with homologous compared to heterologous Plasmodium vivax strains in Papua New Guinean children. Infect. Immun. 77, 4009-4017 (2009).

23. Tsuboi, T. et al. Natural variation within the principal adhesion domain of the Plasmodium vivax Duffy binding protein. Infect. Immun. 62, 5581-5586 (1994).

24. Ntumngia, F. B. \& Adams, J. H. Design and immunogenicity of a novel synthetic antigen based on the ligand domain of the Plasmodium vivax Duffy binding protein. Clin. Vaccin. Immunol. 19, 30-36 (2012).

25. Chen, E., Salinas, N. D., Ntumngia, F. B., Adams, J. H. \& Tolia, N. H. Structural analysis of the synthetic Duffy Binding Protein (DBP) antigen DEKnull relevant for Plasmodium vivax malaria vaccine design. PLoS Negl. Trop. Dis. 9, e0003644 (2015).

26. Schmidt, A. G. et al. Viral receptor-binding site antibodies with diverse germline origins. Cell 161, 1026-1034 (2015).

27. Ekiert, D. C. et al. Antibody recognition of a highly conserved influenza virus epitope. Science 324, 246-251 (2009).

28. Ceravolo, I. P. et al. Naturally acquired inhibitory antibodies to Plasmodium vivax Duffy binding protein are short-lived and allele-specific following a single malaria infection. Clin. Exp. Immunol. 156, 502-510 (2009).

29. VanBuskirk, K. M. et al. Antigenic drift in the ligand domain of Plasmodium vivax Duffy binding protein confers resistance to inhibitory antibodies. J. Infect. Dis. 190, 1556-1562 (2004).

30. Verkoczy, L., Kelsoe, G., Moody, M. A. \& Haynes, B. F. Role of immune mechanisms in induction of HIV-1 broadly neutralizing antibodies. Curr. Opin. Immunol. 23, 383-390 (2011).

31. Orlandi, P. A., Sim, B. K., Chulay, J. D. \& Haynes, J. D. Characterization of the 175-kilodalton erythrocyte binding antigen of Plasmodium falciparum. Mol. Biochem. Parasitol. 40, 285-294 (1990).

32. Klotz, F. W. et al. Binding of Plasmodium falciparum 175-kilodalton erythrocyte binding antigen and invasion of murine erythrocytes requires $\mathrm{N}$-acetylneuraminic acid but not its $\mathrm{O}$-acetylated form. Mol. Biochem. Parasitol. 51, 49-54 (1992).

33. Sim, B. K., Chitnis, C. E., Wasniowska, K., Hadley, T. J. \& Miller, L. H. Receptor and ligand domains for invasion of erythrocytes by Plasmodium falciparum. Science 264, 1941-1944 (1994). 
34. Tolia, N., Enemark, E., Sim, B. \& Joshua-Tor, L. Structural basis for the EBA-175 erythrocyte invasion pathway of the malaria parasite Plasmodium falciparum. Cell 122, 183 (2005).

35. Chen, E. et al. Structural and functional basis for inhibition of erythrocyte invasion by antibodies that target Plasmodium falciparum EBA-175. PLoS Pathog. 9, e1003390 (2013)

36. Salinas, N. D., Paing, M. M. \& Tolia, N. H. Critical glycosylated residues in exon three of erythrocyte glycophorin A engage Plasmodium falciparum EBA-175 and define receptor specificity. mBio 5, 01606-14 (2014).

37. Salinas, N. D. \& Tolia, N. H. A quantitative assay for binding and inhibition of Plasmodium falciparum Erythrocyte Binding Antigen 175 reveals high affinity binding depends on both DBL domains. Protein Expr. Purif. 95, 188-194 (2014).

38. Mayer, D. C., Kaneko, O., Hudson-Taylor, D. E., Reid, M. E. \& Miller, L. H. Characterization of a Plasmodium falciparum erythrocyte-binding protein paralogous to EBA-175. Proc. Natl Acad. Sci. USA 98, 5222-5227 (2001).

39. Narum, D. L., Fuhrmann, S. R., Luu, T. \& Sim, B. K. A novel Plasmodium falciparum erythrocyte binding protein-2 (EBP2/BAEBL) involved in erythrocyte receptor binding. Mol. Biochem. Parasitol. 119, 159-168 (2002).

40. Lobo, C. A., Rodriguez, M., Reid, M. \& Lustigman, S. Glycophorin C is the receptor for the Plasmodium falciparum erythrocyte binding ligand PfEBP-2 (baebl). Blood 101, 4628-4631 (2003).

41. Lin, D. H., Malpede, B. M., Batchelor, J. D. \& Tolia, N. H. Crystal and solution structures of Plasmodium falciparum erythrocyte-binding antigen 140 reveal determinants of receptor specificity during erythrocyte invasion. $J$. Biol. Chem. 287, 36830-36836 (2012).

42. Malpede, B. M., Lin, D. H. \& Tolia, N. H. Molecular basis for sialic acid-dependent receptor recognition by the Plasmodium falciparum invasion protein erythrocyte-binding antigen-140/BAEBL. J. Biol. Chem. 288, 12406-12415 (2013)

43. Lopaticki, S. et al. Reticulocyte and erythrocyte binding-like proteins function cooperatively in invasion of human erythrocytes by malaria parasites. Infect. Immun. 79, 1107-1117 (2011)

44. Persson, K. E. et al. Erythrocyte-binding antigens of Plasmodium falciparum are targets of human inhibitory antibodies and function to evade naturally acquired immunity. J. Immunol. 191, 785-794 (2013).

45. Gilberger, T. W. et al. A novel erythrocyte binding antigen-175 paralogue from Plasmodium falciparum defines a new trypsin-resistant receptor on human erythrocytes. J. Biol. Chem. 278, 14480-14486 (2003).

46. Mayer, D. C. et al. Polymorphism in the Plasmodium falciparum erythrocytebinding ligand JESEBL/EBA-181 alters its receptor specificity. Proc. Natl Acad. Sci. USA 101, 2518-2523 (2004).

47. Maier, A. G., Baum, J., Smith, B., Conway, D. J. \& Cowman, A. F. Polymorphisms in erythrocyte binding antigens 140 and 181 affect function and binding but not receptor specificity in Plasmodium falciparum. Infect. Immun. 77, 1689-1699 (2009).

48. Mayer, D. C. et al. Glycophorin B is the erythrocyte receptor of Plasmodium falciparum erythrocyte-binding ligand, EBL-1. Proc. Natl Acad. Sci. USA 106, 5348-5352 (2009)

49. Ntumngia, F. B. et al. A novel erythrocyte binding protein of Plasmodium vivax suggests an alternate invasion pathway into Duffy-positive reticulocytes. mBio 7, e01261-16 (2016).

50. Gruszczyk, J. et al. Transferrin receptor 1 is a reticulocyte-specific receptor for. Science 359, 48-55 (2018).

51. Orjuela-Sanchez, P. et al. Higher microsatellite diversity in Plasmodium vivax than in sympatric Plasmodium falciparum populations in Pursat, Western Cambodia. Exp. Parasitol. 134, 318-326 (2013).

52. Hostetler, J. B. et al. Independent origin and global distribution of distinct Plasmodium vivax Duffy binding protein gene duplications. PLoS Negl. Trop. Dis. 10, e0005091 (2016)

53. Nicolete, V. C., Frischmann, S., Barbosa, S., King, C. L. \& Ferreira, M. U. Naturally acquired binding-inhibitory antibodies to Plasmodium vivax Duffy binding protein and clinical immunity to malaria in rural Amazonians. J. Infect. Dis. 214, 1539-1546 (2016).

54. Wardemann, H. \& Kofer, J. Expression cloning of human B cell immunoglobulins. Methods Mol. Biol. 971, 93-111 (2013).

55. Tiller, T. et al. Efficient generation of monoclonal antibodies from single human B cells by single cell RT-PCR and expression vector cloning. J. Immunol. Methods 329, 112-124 (2008).

56. Giudicelli, V., Brochet, X. \& Lefranc, M. P. IMGT/V-QUEST: IMGT standardized analysis of the immunoglobulin (IG) and T cell receptor (TR) nucleotide sequences. Cold Spring Harb. Protoc. 2011, 695-715 (2011)

57. Stothard, P. The sequence manipulation suite: JavaScript programs for analyzing and formatting protein and DNA sequences. Biotechniques 28, 1104 (2000).

58. Mirsky, A., Kazandjian, L. \& Anisimova, M. Antibody-specific model of amino acid substitution for immunological inferences from alignments of antibody sequences. Mol. Biol. Evol. 32, 806-819 (2015)

59. Kabsch, W. Xds. Acta Crystallogr. D 66, 125-132 (2010)

60. McCoy, A. J. et al. Phaser crystallographic software. J. Appl. Crystallogr. 40, 658-674 (2007).
61. Marcatili, P., Rosi, A. \& Tramontano, A. PIGS: automatic prediction of antibody structures. Bioinformatics 24, 1953-1954 (2008).

62. Adams, P. D. et al. PHENIX: building new software for automated crystallographic structure determination. Acta Crystallogr. D 58, 1948-1954 (2002).

63. Emsley, P. \& Cowtan, K. Coot: model-building tools for molecular graphics. Acta Crystallogr. D 60, 2126-2132 (2004).

64. Davis, I. W. et al. MolProbity: all-atom contacts and structure validation for proteins and nucleic acids. Nucleic Acids Res. 35, W375-W383 (2007).

65. Krissinel, E. \& Henrick, K. Inference of macromolecular assemblies from crystalline state. J. Mol. Biol. 372, 774-797 (2007).

66. Morin, A. et al. Collaboration gets the most out of software. eLife 2, e01456 (2013).

67. Rangel, G. W. et al. Enhanced ex vivo Plasmodium vivax intraerythrocytic enrichment and maturation for rapid and sensitive parasite growth assays. Antimicrob. Agents Chemother. 62, e02519-17 (2018).

68. Russell, B. et al. A reliable ex vivo invasion assay of human reticulocytes by Plasmodium vivax. Blood 118, e74-e81 (2011).

69. Choe, H. et al. Sulphated tyrosines mediate association of chemokines and Plasmodium vivax Duffy binding protein with the Duffy antigen/receptor for chemokines (DARC). Mol. Microbiol. 55, 1413-1422 (2005).

70. Nóbrega de Sousa, T., Carvalho, L. H. \& Alves de Brito, C. F. Worldwide genetic variability of the Duffy binding protein: insights into Plasmodium vivax vaccine development. PLoS ONE 6, e22944 (2011).

71. Larkin, M. A. et al. Clustal W and Clustal X version 2.0. Bioinformatics 23, 2947-2948 (2007).

72. Waterhouse, A. M., Procter, J. B., Martin, D. M., Clamp, M. \& Barton, G. J. Jalview Version 2-a multiple sequence alignment editor and analysis workbench. Bioinformatics 25, 1189-1191 (2009).

73. Brochet, X., Lefranc, M. P. \& Giudicelli, V. IMGT/V-QUEST: the highly customized and integrated system for IG and TR standardized V-J and V-D-J sequence analysis. Nucleic Acids Res. 36, W503-W508 (2008).

\section{Acknowledgements}

This work was supported by the Intramural Research Program of the National Institute of Allergy and Infectious Diseases, National Institutes of Health, the Extramural Research Program of the National Institute of Allergy and Infectious Diseases, National Institutes of Health (R56 AI080792 to N.H.T.; contract HHSN272201400018C to N.H.T., J.H.A., C.L.K. and M.L.G.; R01 AI064478 to J.H.A., N.H.T. and C.L.K.; and P41 GM103422 to M.L.G.), the Veterans Affairs Research Service (BX001350 to C.L.K.), the Burroughs Wellcome Fund (to N.H.T.) and Fundação de Amparo à Pesquisa do Estado de São Paulo, Brazil (FAPESP, 2009/52729-9 to M.U.F.). V.C.N. is supported by a scholarship from the Conselho Nacional de Desenvolvimento Científico e Tecnológico (CNPq) of Brazil, which also provides a senior researcher scholarship to M.U.F. We thank Y. Colin for the gift of the murine 2C3 anti-DARC. We thank J. Nix and ALS Beamline 4.2.2, supported by contract DE-AC02-05CH11231, the Facility of the Rheumatic Diseases Core Center under award number P30AR048335 and GM/ CA-CAT beamlines 23-ID-D at the Advanced Photon Source, Argonne National Laboratory. We thank C. Nelson for assistance in the analysis of BLI data, A. OdomJohn for the use of a plate reader and J. Patrick Gorres for his assistance in preparing this manuscript for publication.

\section{Author contributions}

Conceptualization: N.H.T., C.L.K. and J.H.A. Methodology: D.U., L.C., Y.H., N.D.S., V.C.N., J.P., C.R. and N.H.T. Validation: D.U., L.C., Y.H., V.C.N., J.P. and C.R. Formal analysis: D.U., L.C., Y.H., N.D.S., V.C.N., J.P. and C.R. Investigation: D.U., L.C., Y.H., V.C.N., J.P. and C.R. Resources: N.H.T., C.L.K., M.L.G., J.H.A., M.U.F. and B.W. Original draft preparation: D.U. and N.H.T. Review and editing of the manuscript: D.U., L.C., Y.H., N.D.S., V.C.N., J.P., C.R., B.W., M.U.F., J.H.A., M.L.G., C.L.K. and N.H.T. Visualization: D.U., Y.H. and N.H.T. Supervision: N.H.T., C.L.K., M.L.G., J.H.A., M.U.F. and B.W. Project administration: N.H.T., C.L.K., M.L.G., J.H.A., M.U.F. and B.W. Funding acquisition: N.H.T., C.L.K., M.L.G., J.H.A., M.U.F. and B.W. S.D. performed experiments and provided results to address reviewer comments during the revision process.

\section{Competing interests}

The authors declare no competing interests.

\section{Additional information}

Supplementary information is available for this paper at https://doi.org/10.1038/ s41564-019-0461-2.

Reprints and permissions information is available at www.nature.com/reprints. Correspondence and requests for materials should be addressed to N.H.T. Publisher's note Springer Nature remains neutral with regard to jurisdictional claims in published maps and institutional affiliations.

(C) The Author(s), under exclusive licence to Springer Nature Limited 2019 


\section{Reporting Summary}

Nature Research wishes to improve the reproducibility of the work that we publish. This form provides structure for consistency and transparency in reporting. For further information on Nature Research policies, see Authors \& Referees and the Editorial Policy Checklist.

\section{Statistical parameters}

When statistical analyses are reported, confirm that the following items are present in the relevant location (e.g. figure legend, table legend, main text, or Methods section).

n/a Confirmed

$\square \bigotimes$ The exact sample size $(n)$ for each experimental group/condition, given as a discrete number and unit of measurement

$\square$ \An indication of whether measurements were taken from distinct samples or whether the same sample was measured repeatedly

$\square$ The statistical test(s) used AND whether they are one- or two-sided

Only common tests should be described solely by name; describe more complex techniques in the Methods section.

Х $\square$ A description of all covariates tested

Х $\square$ A description of any assumptions or corrections, such as tests of normality and adjustment for multiple comparisons

$\triangle$ A full description of the statistics including central tendency (e.g. means) or other basic estimates (e.g. regression coefficient) AND

$\triangle$ variation (e.g. standard deviation) or associated estimates of uncertainty (e.g. confidence intervals)

$\varnothing$ For null hypothesis testing, the test statistic (e.g. $F, t, r$ ) with confidence intervals, effect sizes, degrees of freedom and $P$ value noted

Give P values as exact values whenever suitable.

Х $\square$ For Bayesian analysis, information on the choice of priors and Markov chain Monte Carlo settings

Х $\square$ For hierarchical and complex designs, identification of the appropriate level for tests and full reporting of outcomes

Х $\square$ Estimates of effect sizes (e.g. Cohen's $d$, Pearson's $r$ ), indicating how they were calculated

$\varnothing$ Clearly defined error bars

State explicitly what error bars represent (e.g. SD, SE, CI)

Our web collection on statistics for biologists may be useful.

\section{Software and code}

Policy information about availability of computer code

Data collection Diffraction data was collected using Blu-Ice (ALS). Functional data was incorporated into Excel spreadsheets. Flow cytometry data were collected using CyView v1.6.4.10 and BDdiva v6.1.3.

Data analysis

XDS version January 26, 2018; COOT v0.7 and v0.8; MolProbity v4.3 and v4.4; PDBePISA v1.48; Graphpad Prism v7; FlowJo v10; SBGrid v2017, v2018 and v2019; PHENIX v1.12, v1.13 and v1.14 (includes PHASER); IMGT/V564 Quest v3.4; ClustalW v2.1; JalView v2.10.2

For manuscripts utilizing custom algorithms or software that are central to the research but not yet described in published literature, software must be made available to editors/reviewers upon request. We strongly encourage code deposition in a community repository (e.g. GitHub). See the Nature Research guidelines for submitting code \& software for further information. 
Policy information about availability of data

All manuscripts must include a data availability statement. This statement should provide the following information, where applicable:

- Accession codes, unique identifiers, or web links for publicly available datasets

- A list of figures that have associated raw data

- A description of any restrictions on data availability

Accession codes for the protein structures in the Protein Data Bank will be available before publication. Any reasonable request for data from qualified investigator will be provided.

\section{Field-specific reporting}

Please select the best fit for your research. If you are not sure, read the appropriate sections before making your selection.

$\bigotimes$ Life sciences $\quad \square$ Behavioural \& social sciences $\square$ Ecological, evolutionary \& environmental sciences

For a reference copy of the document with all sections, see nature.com/authors/policies/ReportingSummary-flat.pdf

\section{Life sciences study design}

All studies must disclose on these points even when the disclosure is negative.

Sample size No sampe-size calculation was performed.

Data exclusions Individuals for which cryopreserved peripheral blood mononuclear cells were available were screened for the presence of antibodies to PvDBPII. Those with low antibody titer or did not have functional antibodies were excluded. No other data were excluded. The exclusion criteria were pre-established

Replication All attempts at replication were successful.

Randomization This is predominantly and in vitro study and not a randomized control trial. Randomization is not relevant to the study design.

Blinding Analyzers of the invasion experiments were blinded to the interventions. No other blinding was necessary.

\section{Reporting for specific materials, systems and methods}

Materials \& experimental systems

\begin{tabular}{l}
\hline Involved in the study \\
$\triangle$ Unique biological materials \\
$\square$ Antibodies \\
$\square$ Eukaryotic cell lines \\
$\square$ Animals and other organisms
\end{tabular}

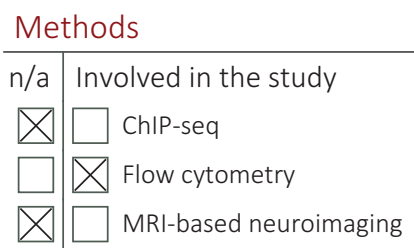

Unique biological materials

Policy information about availability of materials

Obtaining unique materials All unique materials are available from the authors.

\section{Antibodies}

Antibodies used

Human monoclonal antibodies to P. vivax Duffy binding protein were generated as part of the study and characterized in detail. Polyclonal antibodies to PvDBP were generated in previous studies (Grimberg et al. PLoS Med 4, e337 2007; King, C.L. et al. Proc Natl Acad Sci U S A 105, 8363-8 2008). Peroxidase AffiniPure Goat Anti-Human IgG, Fcy fragment specific were obtained from ImmunoResearch Laboratories, Inc. (catalog number 109-035-008) 


\section{Eukaryotic cell lines}

Policy information about cell lines

Cell line source(s)

HEK293-H and HEK293F cell line from ThermoFisher Lifesciences.

Authentication

Cell line was obtained from a commercial source. None of the cell lines have been authenticated.

Mycoplasma contamination

Commonly misidentified lines

(See ICLAC register)

Cell line was obtained from a commercial source and not tested for mycoplasma.

No commonly misidentified cell lines were used.

\section{Human research participants}

Policy information about studies involving human research participants

Population characteristics Patients with clinical malaria attending malaria clinics in Brazil and Cambodia. Patient population ages ranged from 5-60 and included males and females of roughly equal proportions.

Recruitment

Patients were recruited in malaria clinics and offered participation in the study (supply of parasite-infected blood sample) after the study aims and protocol have been described. Written informed consent obtained from all. Participation in the study did not affect treatment practices; malaria treatment was not provided by the research team, but by the clinical staff the malaria outposts. No known self-selection or other biases are expected.

\section{Flow Cytometry}

Plots

Confirm that:

$\square$ The axis labels state the marker and fluorochrome used (e.g. CD4-FITC).

$\bigotimes$ The axis scales are clearly visible. Include numbers along axes only for bottom left plot of group (a 'group' is an analysis of identical markers).

$\bigotimes$ All plots are contour plots with outliers or pseudocolor plots.

$\bigotimes$ A numerical value for number of cells or percentage (with statistics) is provided.

Methodology

Sample preparation

Sample preparation is listed in the Methods section. For the inhibition assays, antigens and antibodies were preincubated together before addition to RBCs and the antigen/antibody mixture added to RBCs and the fluorescence of the samples read. For the invasion studies, deleukocyted cryopreserved iRBCs obtained from Cambodian patients with acute P.vivax malaria were thawed and cultured in IMDM medium. The schizont-infected erythrocytes were enriched using Percoll-KCl, then mixed at a ratio of 1 erythrocyte to 1 with reticulocytes enriched by Percoll from cord blood and labeled with CellTrace Far Red dye (DDAO). Cells were then stained with DNA stain Hoechst 33342.

Instrument

BDFacsCanto machine, Partec cube 8

Software

BDdiva, CyView, FlowJo

Cell population abundance

For the inhibition assays, populations were not sorted as only the MFI was analyzed in these experiments. For the invasion studies, FACS analysis was performed on 40,000 cells/acquisition without cell sorting.

Gating strategy

For the inhibition assays, no gates were conducted as only the MFI was analyzed in these experiments. For the invasion studies, red blood cells (the only cell type) are gated on FSC/SSC. DDAO-positive cells are then gated on FL5 axis (2 log difference with negative cells). Hoechst positive DDAO+ cells are gated on FL4 axis. Boundary between Hoechst+ and - cells is defined at the bottom of the peak of negative cells on the FL4 histogram.

Tick this box to confirm that a figure exemplifying the gating strategy is provided in the Supplementary Information. 